\title{
ATCA observations of the galaxy cluster Abell 3921
}

\section{Radio emission from the central merging sub-clusters}

\author{
C. Ferrari ${ }^{1}$, R. W. Hunstead ${ }^{2}$, L. Feretti ${ }^{3}$, S. Maurogordato ${ }^{4}$, and S. Schindler ${ }^{1}$ \\ 1 Institut für Astro- und Teilchen Physik, Universität Innsbruck, Technikerstraße 25, 6020 Innsbruck, Austria \\ e-mail: chiara.ferrari@uibk.ac.at \\ 2 School of Physics, University of Sydney, NSW 2006, Australia \\ 3 Istituto di Radioastronomia - INAF, via Gobetti 101, 40129 Bologna, Italy \\ ${ }^{4}$ Laboratoire Cassiopée, CNRS, Observatoire de la Côte d'Azur, BP 4229, 06304 Nice Cedex 4, France
}

Received 28 February 2006 / Accepted 29 June 2006

\section{ABSTRACT}

\begin{abstract}
Context. We present the analysis of our 13 and $22 \mathrm{~cm}$ ATCA observations of the central $\sim 18 \times 15 \operatorname{arcmin}^{2}$ region of the merging galaxy cluster A3921 $(z=0.094)$.

Aims. We investigated the effects of the major merger between two sub-clusters on the star formation (SF) and radio emission properties of the confirmed cluster members.

Methods. The origin of SF and the nature of radio emission in cluster galaxies was investigated by comparing their radio, optical and $\mathrm{X}$-ray properties. We also compared the radio source counts and the percentage of detected radio galaxies with literature data.

Results. We detected 17 radio sources above the flux density limit of $0.25 \mathrm{mJy} /$ beam in the central field of A3921, among which 7 are cluster members. 9 galaxies with star-forming optical spectra were observed in the collision region of the merging sub-clusters. They were not detected at radio wavelengths, giving upper limits for their star formation rate significantly lower than those typically found in late-type, field galaxies. Most of these star-forming objects are therefore really located in the high density part of the cluster, and they are not infalling field objects seen in projection at the cluster centre. Their SF episode is probably related to the cluster collision that we observe in its very central phase. None of the galaxies with post-starburst optical spectra was detected down our $2 \sigma$ flux density limit, confirming that they are post-starburst and not dusty star-forming objects. We finally detected a narrow-angle tail (NAT) source associated with the second brightest cluster galaxy (BG2), whose diffuse component is a partly detached pair of tails from an earlier period of activity of the BG2 galaxy.
\end{abstract}

Key words. galaxies: clusters: general - galaxies: clusters: individual: Abell 3921 - radio continuum: galaxies

\section{Introduction}

In the past 25 years, optical observations of clusters have revealed an evolution in galaxy properties with redshift. The fraction of star-forming and post-star-forming cluster objects significantly increases with $z$, ranging from $\sim 1-2 \%$ in the local Universe (Dressler 1987) to $\geq 30 \%$ at $z \sim 0.3-0.5$ (Dressler et al. 1999). In 1978, Butcher \& Oemler reported a strong evolution of the mean galaxy colour with redshift, from red to blue with increasing $z$. A debate on the physical origin of this effect began: the observed change of galaxy colours with redshift could be simply due to a passive evolution of infalling field galaxies, or it could be strongly affected by environmental effects. In 1983 Dressler \& Gunn pointed out for the first time that in fact the blue colour of the population detected by Butcher \& Oemler was the result of star formation (SF) activity. Since then, many different studies have tried to explain the origin of the observed evolution in the SF history of cluster galaxies. The different physical mechanisms that have been proposed may either trigger or weaken SF within clusters (e.g. Dressler \& Gunn 1983; Evrard 1991; Bekki 1999; Fujita et al. 1999).

The hierarchical model of structure formation predicts the formation and evolution of galaxy clusters through mergers of less massive systems. Due to the large energies involved in cluster-cluster collisions, a merging event can enhance the efficiency of the physical mechanisms responsible for the evolution of the galaxy star formation rate (SFR). The first observational evidence for a correlation between cluster mergers and SF activity came from the optical analysis of the Coma cluster, where an excess of star-forming and post-star-forming galaxies was observed in the collision region between the main cluster and a group of galaxies (Caldwell et al. 1993). While in several other non-relaxed clusters the collision between two or more clumps seems to have increased the SFR of the galaxies (e.g. Gavazzi et al. 2003), other analyses show the opposite trend (e.g. Baldi et al. 2001). Therefore, the net role played by the merging event on SF still has to be fully understood. To answer this question, multi-wavelength (optical, X-ray, radio and/or IR) observations of galaxy clusters are essential, as they allow us to determine in detail both the dynamical state of the observed systems and the SF properties of cluster members (e.g. Dwarakanath \& Owen 1999; Owen et al. 1999; Venturi et al. 2000, 2001, 2002; Duc et al. 2002; Miller et al. 2003; Rizza et al. 2003; Giacintucci et al. 2004; Owen et al. 2005a,b).

Abell 3921 is a $R=2$, BM II Abell cluster at $z=0.094$ (Katgert et al. 1996, 1998). Its perturbed morphology, first revealed by ROSAT and Ginga observations (Arnaud et al. 1996), has been recently confirmed by new optical (multiobject spectroscopy and VRI deep imaging, Ferrari et al. 2005, F05 in the following) and X-ray analyses (XMM observations, 
Belsole et al. 2005, B05 in the following). Two dominant clumps of galaxies with a distorted morphology and a mass ratio of $\sim 5$ have been detected: a main cluster centred on the Brightest Cluster Galaxy (BCG) (A3921-A), and an NW subcluster (A3921-B) hosting the second brightest cluster member. The comparison of the optical and X-ray properties of A3921 (i.e. dynamical and kinematic properties of the cluster, optical and X-ray morphology, features in the ICM density and temperature maps) suggests that A3921-B is probably tangentially traversing the main cluster along the $\mathrm{SW} / \mathrm{NE}$ direction. The two sub-clusters are observed in the central phase of their merging process $\left(t_{\text {coll }} \sim \pm 0.3 \mathrm{Gyr}\right)$, with a collision axis approximately on the plane of the sky (F05; B05). A comparison of the metallicity distribution in A3921 with numerical simulations refined the reconstructed merging scenario, suggesting that we are observing the very central phase of the clusters' interaction, just before the first core-core encounter (Kapferer et al. 2006).

From spectral features of galaxies belonging to A3921, the SF properties of confirmed cluster members have also been studied (F05). Substantial fractions of both emission-line and poststar-forming objects (so called k+a's) have been detected, comparable to those measured at intermediate redshifts. Most of the $\mathrm{k}+\mathrm{a}$ galaxies in A3921 have fainter magnitudes than the poststar-forming objects detected in higher redshift clusters ("downsizing effect", e.g. Poggianti et al. 2004). Since emission-line galaxies in A3921 share neither the kinematics nor the projected distribution of the passive cluster members, and they are mostly concentrated in the region between the two sub-clusters, F05 concluded that the ongoing merger may have triggered a SF episode in the galaxies located inside or around the collision region, where a hot compression bar has been detected in the ICM temperature distribution (B05). The on-going merger could also be responsible for the high fraction of star-forming and post-starforming objects in this low redshift cluster.

These results motivated new high sensitivity radio observations of A3921 with the Australia Telescope Compact Array (ATCA), in order to shed light on the possible connection between the detected merger and the SF and AGN activity in cluster galaxies. This paper deals with the analysis of the radio emission in the central region of the cluster, where we detected the major merger between the main sub-clusters A3921-A and A3921-B. Section 2 briefly describes the observations and the data reduction. In Sects. 3 and 4 we analyse the radio emission from the A3921 central region and from the confirmed cluster members. The nature of the peculiar radio source associated with the second brightest cluster galaxy is discussed in Sect. 5. The main results and their interpretation are summarized in Sect. 6.

As in F05 and B05, throughout the paper we assume $H_{0}=$ $75 \mathrm{~km} \mathrm{~s}^{-1} \mathrm{Mpc}^{-1}, \Omega_{\mathrm{m}}=0.3, \Omega_{\Lambda}=0.7$. In this cosmology 1 arcmin corresponds to $\sim 0.097 h_{75}{ }^{-1} \mathrm{Mpc}$.

\section{Observations and data reduction}

We observed the central $\sim 18 \times 15 \operatorname{arcmin}^{2}\left(\sim 1.8 \times 1.5 \mathrm{Mpc}^{2}\right)$ field of A3921 covered by our previous optical observations with the Australia Telescope Compact Array (ATCA) at $22 \mathrm{~cm}(v=$ $1.344 \mathrm{GHz})$ and $13 \mathrm{~cm}(v=2.368 \mathrm{GHz})$ simultaneously. The ATCA comprises five $22 \mathrm{~m}$ antennas placed on a $3 \mathrm{~km}$ railway track, running east-west, and an isolated sixth antenna, located $3 \mathrm{~km}$ further to the west. There are several stations on which the antennas can be placed, thus allowing a selection of extended or compact configurations with a range of different spacings. The configurations used here, which together gave reasonably uniform uv coverage, are given in Table 1 , where the minimum and
Table 1. Minimum and maximum spacings between antennas in the East-West array configurations used for our ATCA observations.

\begin{tabular}{lcc}
\hline \hline Configuration & \multicolumn{2}{l}{ Baselines (metres) } \\
& Min & Max \\
\hline $6 \mathrm{~A}$ & 337 & 5939 \\
$6 \mathrm{C}$ & 153 & 6000 \\
$750 \mathrm{C}$ & 46 & 5020 \\
$1.5 \mathrm{D}$ & 107 & 4439 \\
\hline
\end{tabular}

Table 2. r.m.s. noise in the 1.344 and $2.368 \mathrm{GHz}$ images. The FWHM of the restoring beam is shown in the third column.

\begin{tabular}{ccc}
\hline \hline $\begin{array}{c}v \\
\mathrm{GHz}\end{array}$ & $\begin{array}{c}\text { r.m.s. noise } \\
\text { mJy/beam }\end{array}$ & $\begin{array}{c}b \\
\operatorname{arcsec}\end{array}$ \\
\hline 1.344 & 0.049 & 12.0 \\
2.368 & 0.052 & 8.0 \\
2.368 & 0.053 & 12.0 \\
\hline
\end{tabular}

maximum spacings are indicated. Observations were performed in continuum mode with a bandwidth of $128 \mathrm{MHz}$ in May, July, November and December 2004. A uniform sensitivity radio map of the central $18 \times 15 \operatorname{arcmin}^{2}$ of A3921 was obtained by using the mosaicing facility of the ATCA. The central cluster field was covered with eight overlapping pointings separated by 10 arcmin (i.e. half the primary beam width at $13 \mathrm{~cm}$ ). To obtain good hour angle coverage, the eight pointings were observed in sequence every $160 \mathrm{~s}$. The cycle, including calibration, was repeated for $12 \mathrm{~h}$ for each observing run. B1934-638 and B2205-636 were used as primary and secondary calibrators. The largest structure detectable by these observations corresponds to $\sim 16$ and 10 arcmin at 22 and $13 \mathrm{~cm}$ respectively.

Data reduction was carried out following the standard procedure (calibration, Fourier inversion, clean and restore) with the MIRIAD package. We reduced and imaged each pointing separately. The eight cleaned maps at 22 and $13 \mathrm{~cm}$ were finally mosaiced (i.e. linearly combined) with the MIRIAD task LINMOS, using the taper option of this program, which corrects for primary beam attenuation avoiding excessive noise amplification at the edge of the mosaic field (Sault et al. 1996). The noise levels in the final maps are given in Table 2. We also obtained a spectral index $\left(\alpha_{13}^{22}\right)$ map by combining the images at $1.344 \mathrm{GHz}$ and $2.368 \mathrm{GHz}$ with the AIPS task COMB. For this purpose, maps at the two frequencies were made with the same beam (i.e. $12 \times 12$ arcsec) and the same uv-range (i.e. 0.354$30 \mathrm{k} \lambda$ ). The output image was blanked whenever the intensity of the corresponding pixel was lower than twice the map noise in either image.

\section{Radio emission from the central field of A3921}

\subsection{The sample}

Within the central $\sim 18 \times 15 \operatorname{arcmin}^{2}$ of $\mathrm{A} 3921$ we found 17 radio sources above a peak flux density limit of 5 times the r.m.s. noise in the $1.344 \mathrm{GHz}$ map. The dynamic range is of the order of $1000: 1$, so the local noise level around strong sources may be higher than that given in Table 2 .

The source list is reported in Table 3, where we give:

- Cols. (1), (2): radio ID and name of detected sources. We used the prefix "FHF2006" (i.e. initials of the first three authors and year of publication) for the names of sources not present in any previous radio survey; 
Table 3. List of $5 \sigma$ sources in the central field $\left(\sim 18 \times 15 \operatorname{arcmin}^{2}\right)$ of A3921. Their position, integrated intensity at 22 and $13 \mathrm{~cm}$, mean spectral index and morphology ("r" = resolved, " $u$ " = unresolved) are given. Errors are indicated in brackets. The colon indicates a highly uncertain value. Spectral index is not given for highly uncertain $13 \mathrm{~cm}$ fluxes. For resolved sources, the measured size was obtained from a Gaussian fit to the $22 \mathrm{~cm}$ image, except for R5 (SUMSS J224857-642059; see text).

\begin{tabular}{|c|c|c|c|c|c|c|c|c|}
\hline $\begin{array}{l}\mathrm{ID}_{\mathrm{R}} \\
\#\end{array}$ & Source name & $\begin{array}{c}\mathrm{RA}_{\mathrm{J} 2000}(\mathrm{err}) \\
\mathrm{h} \mathrm{m} \mathrm{s}(s)\end{array}$ & 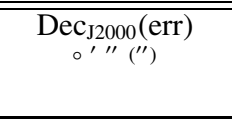 & $\begin{array}{c}S_{22 \mathrm{~cm}}(\mathrm{err}) \\
\mathrm{mJy}\end{array}$ & $\begin{array}{c}S_{13 \mathrm{~cm}}(\mathrm{err}) \\
\mathrm{mJy}\end{array}$ & 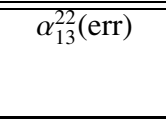 & Mor. & $\begin{array}{c}\begin{array}{l}\text { Size } \\
\text { " } \times \text { " } \\
\text { deg }\end{array} \\
\end{array}$ \\
\hline R1 & SUMSS J224834-642037 & $224835.24(0.03)$ & $-642037.3(0.5)$ & $11.79(0.14)$ & $8.11(0.14)$ & $-0.66(0.04)$ & $\mathrm{u}$ & - \\
\hline $\mathrm{R} 2$ & FHF2006 J224839-641921 & 2248 39.74(0.13) & $-641921.7(1.1)$ & $0.36(0.08)$ & $0.31(0.11)$ & -0.3 : & $\mathrm{u}$ & - \\
\hline R3 & FHF2006 J224848-642311 & $224848.74(0.06)$ & $-642311.4(0.6)$ & $1.80(0.05)$ & $1.32(0.05)$ & $-0.55(0.08)$ & $\mathrm{r}$ & $\begin{array}{c}16 \times 14, \\
\text { p.a. }=102\end{array}$ \\
\hline $\mathrm{R} 4$ & FHF2006 J224902-642743 & $224902.60(0.04)$ & $-642743.9(0.5)$ & $0.37(0.06)$ & $0.21(0.07)$ & -1.0 & $\mathrm{u}$ & - \\
\hline R5 & SUMSS J224857-642059 & $224904.63(0.03)$ & $-642036.3(0.5)$ & $43.80(0.44)$ & $13.95(0.15)$ & $-2.02(0.03)$ & $\mathrm{r}$ & $\begin{array}{c}105 \times 99, \\
\text { p.a. }=0\end{array}$ \\
\hline R6 & FHF2006 J224904-641816 & $224904.98(0.04)$ & $-641816.3(0.5)$ & $1.90(0.07)$ & $1.29(0.07)$ & $-0.68(0.11)$ & $\mathrm{r}$ & $\begin{array}{l}16 \times 12, \\
\text { p.a. }=16\end{array}$ \\
\hline R7 & PMN J2249-6415 & $224912.73(0.03)$ & $-641534.0(0.5)$ & $133.20(1.35)$ & 81.02(0.89) & $-0.88(0.03)$ & $\mathrm{u}$ & - \\
\hline R8 & FHF2006 J225007-642408 & $225007.00(0.05)$ & $-642408.9(0.5)$ & $0.88(0.09)$ & $0.59(0.43)$ & -0.7 : & $\mathrm{u}$ & $\begin{array}{l}- \\
-\end{array}$ \\
\hline R9 & SUMSS J225007-642439 & $225007.72(0.03)$ & $-642440.9(0.5)$ & $63.82(0.64)$ & $42.55(0.43)$ & $-0.72(0.03)$ & $\mathrm{r}$ & $\begin{array}{l}21 \times 12, \\
\text { p.a. }=81\end{array}$ \\
\hline $\mathrm{R} 10$ & FHF2006 J225013-641935 & $225013.64(0.07)$ & $-641935.3(0.6)$ & $0.82(0.05)$ & $0.33(0.05)$ & $-1.61(0.29)$ & $\mathrm{r}$ & $\begin{array}{c}15 \times 12, \\
\text { p.a. }=124\end{array}$ \\
\hline R11 & FHF2006 J225026-642819 & $225026.41(0.04)$ & $-642819.8(0.5)$ & $2.18(0.05)$ & $1.20(0.05)$ & $-1.06(0.08)$ & $\mathrm{r}$ & $\begin{array}{l}14 \times 13, \\
\text { p.a. }=27\end{array}$ \\
\hline $\mathrm{R} 12$ & FHF2006 J225035-642542 & $225035.61(0.15)$ & $-642542.8(1.3)$ & $0.25(0.07)$ & 0.2 : & - & $\mathrm{u}$ & - \\
\hline $\mathrm{R} 13$ & FHF2006 J225036-641608 & $225036.52(0.11)$ & $-641608.4(0.7)$ & $0.33(0.09)$ & 0.3: & - & $\mathrm{u}$ & - \\
\hline $\mathrm{R} 14$ & FHF2006 J225038-642020 & $225038.24(0.11)$ & $-642020.1(1.2)$ & $0.80(0.05)$ & $0.64(0.05)$ & $-0.40(0.19)$ & $\mathrm{r}$ & $\begin{array}{l}24 \times 14, \\
\text { p.a. }=7\end{array}$ \\
\hline $\mathrm{R} 15$ & SUMSS J225046-641658 & $225046.60(0.03)$ & $-641659.4(0.5)$ & $21.04(0.23)$ & $12.89(0.37)$ & $-0.87(0.05)$ & $\mathrm{u}$ & $\begin{array}{l}- \\
-\end{array}$ \\
\hline $\mathrm{R} 16$ & FHF2006 J225050-642720 & $225050.59(0.05)$ & $-642720.0(0.6)$ & $0.82(0.08)$ & $0.4:$ & - & $\mathrm{u}$ & - \\
\hline R17 & FHF2006 J225052-642443 & $225052.57(0.11)$ & $-642443.8(0.9)$ & $0.30(0.07)$ & $0.4:$ & - & $\mathrm{u}$ & $\begin{array}{l}- \\
- \\
\end{array}$ \\
\hline
\end{tabular}

- Cols. (3), (4): right ascension and declination (J2000.0) with errors;

- Cols. (5)-(7): integrated intensity at 22 and $13 \mathrm{~cm}$ and spectral index with errors ${ }^{1}$;

- Col. (8): radio morphology ("resolved" or "unresolved");

- Col. (9): measured angular size of the source, if resolved.

The total flux density and the position of the flux density peak at $22 \mathrm{~cm}$ of the irregular resolved source R5 (SUMSS J224857-642059) were calculated using the AIPS task TVSTAT. Its size was estimated using the verb TVDIST. For all the other regular (circular/elliptical) sources the position, integrated intensity and measured size were obtained with a Gaussian fit using the AIPS task IMFIT. The errors in Gaussian fits were taken into account following Condon (1997). We fitted six free parameters (amplitude $A$, central position $\left(x_{0}, y_{0}\right)$, major and minor axes $\left(\theta_{\mathrm{M}}, \theta_{\mathrm{m}}\right)$, and position angle $\left.\beta\right)$. We retained the fitted total flux density $\left(S_{\mathrm{t}}\right)$ only for resolved sources. In the other cases the quoted total flux density corresponds to the corrected amplitude, i.e. the fitted amplitude $\left(S_{\mathrm{p}}\right)$ multiplied by the square root of the

${ }^{1}$ The radio spectral index $\alpha$ is defined such as $S_{v} \propto v^{\alpha}$. ratio of the fitted size of the source $\left(\theta_{\mathrm{M}} \theta_{\mathrm{m}}\right)$ and the beam size $\left(\theta_{\mathrm{M}}^{*} \theta_{\mathrm{m}}^{*}\right.$, Eq. (29) of Condon 1997):

$S_{\mathrm{t}}=S_{\mathrm{p}}\left(\frac{\theta_{\mathrm{M}} \theta_{\mathrm{m}}}{\theta_{\mathrm{M}}^{*} \theta_{\mathrm{m}}^{*}}\right)^{1 / 2}$.

Unresolved or barely resolved sources were identified at $22 \mathrm{~cm}$ as those whose fitted size is within $1 \sigma$ of the map beam size, and/or:

$\frac{S_{\mathrm{t}}-\Delta S_{\mathrm{t}}}{S_{\mathrm{p}}+\Delta S_{\mathrm{p}}}<1$

where $S_{\mathrm{t}}$ and $S_{\mathrm{p}}$ are the fitted integrated and peak fluxes, and $\Delta S_{\mathrm{t}(\mathrm{p})}$ are the associated errors:

$\Delta S_{\mathrm{t}(\mathrm{p})}=\sqrt{\sigma_{\mathrm{Noise}^{2}}+\left(0.01 S_{\mathrm{t}(\mathrm{p})}\right)^{2}}$.

The errors in position $(\Delta \alpha(\delta))$ quoted in Table 3 were estimated as the quadratic combination of the formal IMFIT errors and a nominal $0.5^{\prime \prime}$ calibration uncertainty. The errors in flux density were similarly obtained as the quadratic combination of the r.m.s. noise of the radio map and the formal uncertainty in the total flux density. This latter error was assumed as in Eq. (3) for 
resolved sources (i.e. $0.01 \mathrm{~S}_{\mathrm{t}}$ ), and estimated through error propagation from Eq. (1) for unresolved sources.

\subsection{Comparison with background counts}

As mentioned in the previous section, we counted 17 radio sources with $S_{22 \mathrm{~cm}} \geq 0.25 \mathrm{mJy}$ in the central area $\left(0.075 \mathrm{deg}^{2}\right)$ of A3921. We then compared this number of radio sources with field counts, in order to test the possible presence of an excess of radio emission in the region of the cluster hosting the main merger between A3921-A and A3921-B. The sensitivity of the radio image analysed in this paper is uniform to the $5 \sigma$ flux density limit $(0.25 \mathrm{mJy} / \mathrm{beam})$ due to the overlapping of the 8-pointings of the mosaic in the central region of A3921.

Our results were compared with those of Hopkins et al. (2003). By integrating their polynomial fit for source counts, 25 field radio sources are expected down to the flux limit of our radio observations. Assuming Poissonian noise for our counts, we found a slight deficit of radio sources in our cluster field at $\sim 2 \sigma$ level. Figure 2 shows the radio flux density distribution in A3921 and in the field (Hopkins et al. 2003). At $~ 2 \sigma$ level, there is either a possible excess of radio sources in the field in the lowest flux density bin $(0.25-0.50 \mathrm{mJy})$, or the A3921 counts are incomplete at the faint end. Based on a two-sided Kolmogorov-Smirnov test, the probability that the two distributions are taken from the same parent population is $14 \%$, so the test is not conclusive.

\subsection{Radio-optical identification}

Using our VRI catalogue (WFI data, F05), we found optical identifications for 14 of the 17 radio sources above $5 \sigma$. They are listed in Table 4, which contains:

- Col. (1): radio ID based on Table 3;

- Col. (2): optical ID based on Table A of F05 (when available);

- Cols. (3), (4): optical coordinates;

- Col. (5): offset $\Delta_{\mathrm{r}-\mathrm{o}}$ between the radio and optical coordinates;

- Col. (6): parameter $\mathcal{R}=\Delta_{\mathrm{r}-\mathrm{o}} /\left(\sigma_{\mathrm{o}}^{2}+\sigma_{\mathrm{r}}^{2}\right)^{1 / 2}$, where $\sigma_{\mathrm{r}}$ (given in Table 3$)$ and $\sigma_{\mathrm{o}}(0.1 \mathrm{arcsec})$ are the radio and optical position errors respectively;

- Cols. (7)-(9): $V, R$ and $I$-band magnitudes (WFI observations, F05);

- Col. (10): redshift of the source (when available);

- Col. (11): Log of the absolute spectral luminosity at $1.344 \mathrm{GHz}(\mathrm{W} / \mathrm{Hz})$, estimated when the redshift is available from $L=4 \pi D_{L}^{2} S_{1.344 \mathrm{GHz}}(1+z)^{-(1+\alpha)}$ (Petrosian \& Dickey 1973), where $\alpha$ is the spectral index of the source.

The 14 identified radio sources are shown in Fig. 3 with $22 \mathrm{~cm}$ radio contours overlaid on the $R$-band WFI images. We considered as reliable identifications all those with $\mathcal{R} \leq 3$. For the extended source $\mathrm{R} 9$, which shows a radio-optical offset along the extension of the radio emission and has $\mathcal{R}>3(=12.40)$, we relied on direct inspection by eye rather than the value of $\mathcal{R}$. We concluded that R9 is associated with the bright galaxy $(R \sim 15)$ on the western side of the radio emission (see Fig. 3), and that it has a head-tail radio morphology along the West/East direction. A weak steepening of the spectrum is observed on the spectral index map of the source moving from the head towards East. R9 is a confirmed cluster member, located in the central, high-density region of A3921. Its radio morphology is therefore probably due to the ram-pressure ${ }^{2}$ exerted on the radio emitting plasma by the dense ICM, through which the galaxy is moving towards the cluster centre (in the East/West direction). The extremely peculiar morphology of the radio source R5, associated with the second BCG, will be discussed in detail in Sect. 5.

We also noticed that two sources (R5 and R10) have steep spectral indexes $(\leq-1.50)$. R5 is a confirmed cluster member, while we do not have redshift information for R10. Based on its magnitude and morphology, R10 is probably a cluster galaxy: it occupies the region of $\mathrm{A} 3921$ red-sequence on the colour magnitude diagrams $\left((V-R)_{\mathrm{AB}}\right.$ vs. $R_{\mathrm{AB}}$ and $(R-I)_{\mathrm{AB}}$ vs. $\left.I_{\mathrm{AB}}, \mathrm{F} 05\right)$ and its morphology and size are very similar to its projected neighbour (see Fig. 3) which is at the cluster redshift (Katgert et al. 1996, 1998). The two radio sources with steepest spectra are therefore very likely cluster members. Their steep radio spectra may be related to the pressure of the ICM, which prevents adiabatic expansion (e.g. Murgia et al. 2004).

\section{Star-forming galaxies and AGNs in the A3921 central field}

Of the 14 radio sources with optical identification, spectroscopic redshifts are available for seven of the host galaxies; of these, five lie at the cluster redshift. Due to their colour and size, we considered the two additional radio identifications without redshift information (R10 (see Sect. 3.3) and R11) as likely cluster member candidates. This section concentrates upon these 7 radio emitting cluster members (confirmed or candidate) and upon the star-forming and post-star-forming galaxies identified in the optical (F05).

The optical spectroscopic analysis of the A3921 central field revealed the presence of 24 galaxies with spectral signatures of recent or ongoing SF (F05). The spectral classification was based on the presence and strength of the $\mathrm{H}_{\delta} \lambda 4101$ and [OII] $\lambda 3727$ lines, observed in absorption and emission, respectively. Galaxies presenting an intermediate and strong $\mathrm{H}_{\delta}$ but no [OII] emission were classified as post-star-burst/poststar-forming ( $\mathrm{k}+\mathrm{a}, 13$ objects). The presence of [OII] emission was considered as a possible hint for ongoing SF. F05 found 11 emission line galaxies that, based on the MORPHS classification (Dressler et al. 1999; Poggianti et al. 1999), were divided into: i) two e(b)-type objects (i.e. possible star-burst galaxies, Poggianti et al. 1999), with strong [OII] emission $(E W<-5 \AA)$, ii) four e(c)-type galaxies, with weak Balmer absorption and weak to moderate [OII] emission, classified as spirals forming stars at a constant rate by Poggianti et al. (1999); and iii) five e(a)-type objects, with strong $\mathrm{H}_{\delta}$ absorption and measurable [OII] emission, suggested to be either dusty star-burst or post-star-burst galaxies with some residual SF (Poggianti et al. 1999). They are given in Table 5 (upper part). Most of the emission line galaxies are concentrated in the region of A3921-B and in between the two main sub-clusters A and B (see Fig. 22 in F05 and top panel of Fig. 1 in the present paper), suggesting that the ongoing merging event in A3921 could have triggered their SF/star-burst episode.

However, the [OII] emission line as an estimator of SF is fraught with problems (e.g. Kennicutt 1992), the most important of which is that it suffers from strong dust extinction. As a consequence, the SF activity can be strongly underestimated (or even not detected) if only determined with [OII]. Low-power radio emission from galaxies is, on the contrary, an extinctionfree tracer of SF. Moreover, in our case the spectral range

\footnotetext{
${ }^{2} P_{\text {ram }} \propto \rho_{\text {ICM }} * v_{\text {rel }}^{2}$, where $\rho_{\text {ICM }}$ is the density of the intra-cluster medium, and $v_{\text {rel }}$ is the relative velocity between the ICM and the galaxy.
} 


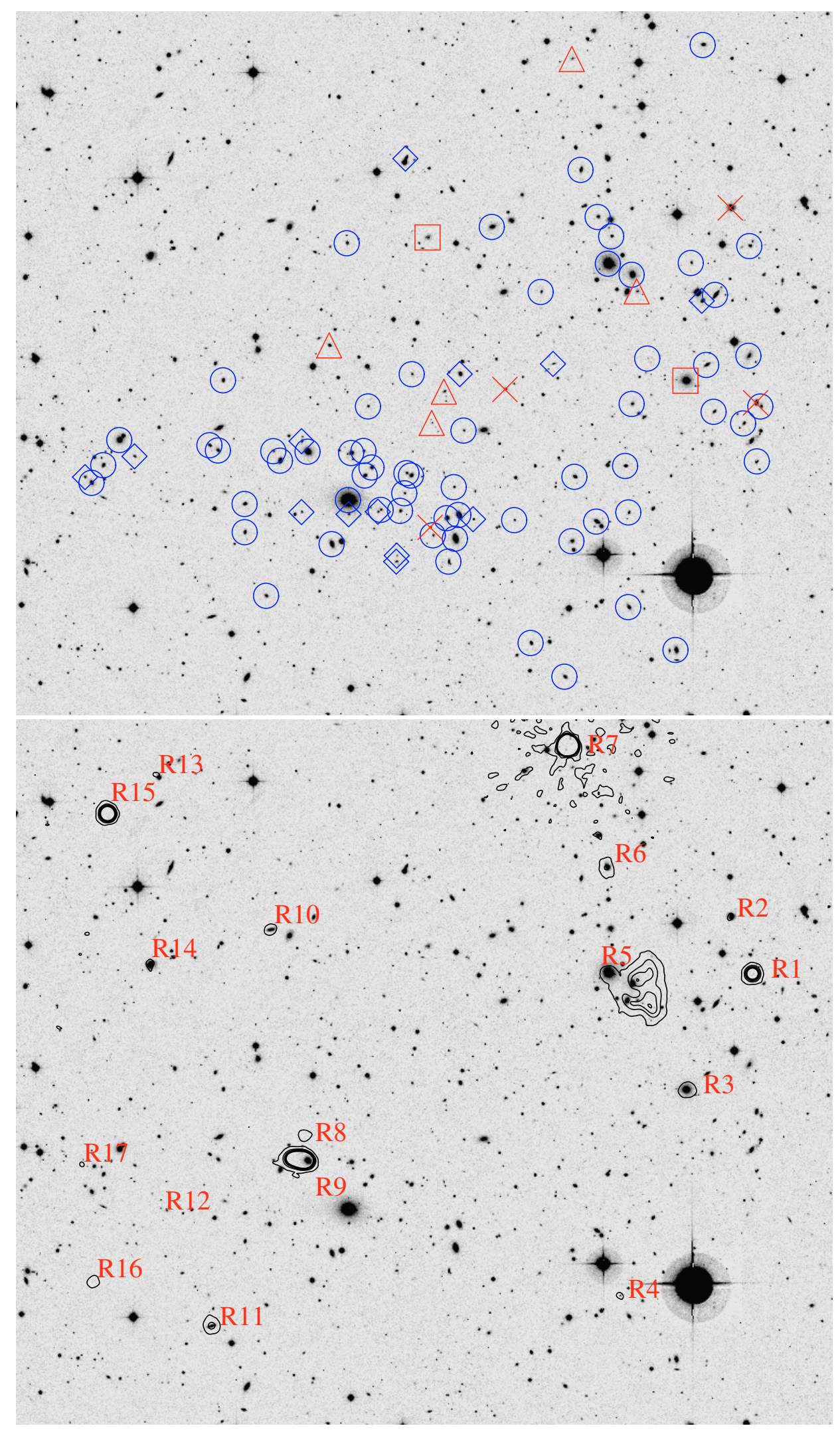

Fig. 1. Top: $R$-band image of the central field $\left(\sim 18 \times 15 \operatorname{arcmin}^{2}\right)$ of A3921. Overlaid symbols correspond to A3921 confirmed cluster members. Based on their spectral properties, galaxies were classified as old passively evolving ellipticals (circles), e(b)'s (squares), e(a)'s (triangles), e(c)'s (crosses), k+a's (diamonds) (see text and F05 for more details). Bottom: $1.344 \mathrm{GHz}$ radio contours are overlaid on the $R$-band central field of A3921. Contour levels start at $0.245 \mathrm{mJy} / \mathrm{beam}(5 \times$ r.m.s. of the $22 \mathrm{~cm}$ map), with linear steps of $1.000 \mathrm{mJy} / \mathrm{beam}$ up to $4.245 \mathrm{mJy} / \mathrm{beam}$. The radio ID of each source is also shown (see Table 3 ). 


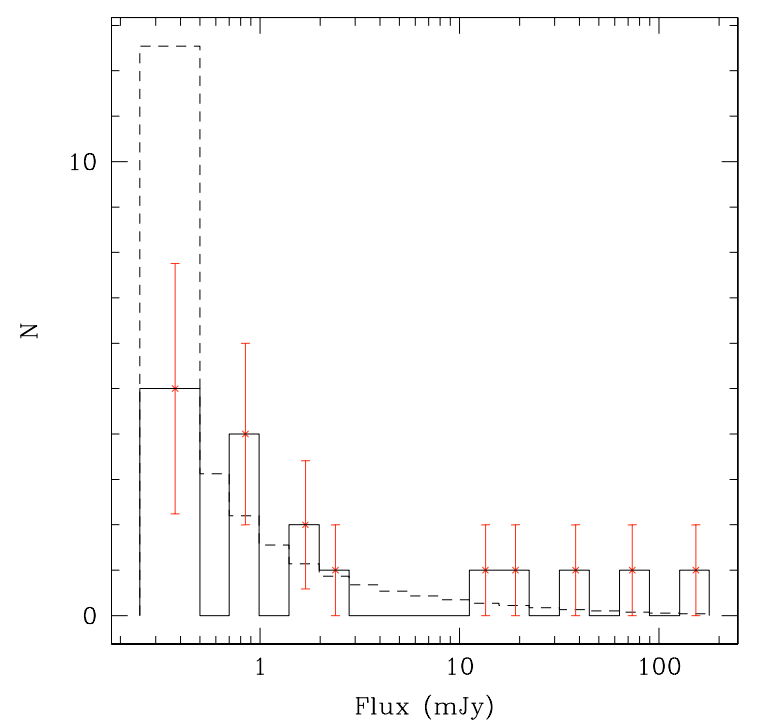

Fig. 2. Flux density distribution of the radio sources detected in A3921 (solid line) and in the field (dashed line). The field counts, derived from Hopkins et al. (2003), have been normalised to the sky area considered in this paper $\left(0.075 \mathrm{deg}^{2}\right) 1 \sigma$ error bars associated with our counts are also shown (assuming Poissonian noise).

covered by the optical observations ( 3000-6000 $\mathrm{A})$ was not sufficient to discriminate between star-forming/bursting objects and AGNs among the emission-line galaxies. In order to test if the merging event has affected the star formation history (SFH) of A3921 galaxies we still need to:

- divide emission line galaxies into star-forming/bursting objects and AGN;

- verify that the emission line galaxies are really located in the collision region and not infalling field galaxies seen in projection at the cluster centre. In such a case the merging event would not be affecting either the SF or the nuclear activity of these objects;

- test the hypothesis that all the detected k+a-type objects are in a post-star-bursting/post-star-forming phase. $\mathrm{k}+\mathrm{a}$ galaxies could also be strongly obscured star-burst galaxies (e.g. Smail et al. 1999; Poggianti \& Wu 2000), in which the [OII] line is totally extinguished. In such a case, star-bursting galaxies would not be concentrated only in the collision region of A3921, but spread all over the cluster (see Fig. 18 of F05). The connection between the sub-clusters' merger and the on-going star-burst episode would obviously be less probable.

We thus combined the optical and radio observations in order to measure the SFR of the cluster members independently from dust extinction and classify the active galaxies of the A3921 central field either as star-forming/bursting objects or AGNs.

\subsection{Emission-line galaxies: optical/radio comparison}

\subsubsection{Optical and radio SFR}

To obtain star-formation rates from radio luminosities, we used the relation from Condon (1992), which was estimated assuming solar abundances and a Salpeter initial mass function (IMF) above $5 M_{\odot}$. In order to estimate the SFR of stars more massive than $0.1 M_{\odot}$, we divided Condon's relation by 0.18 (e.g. Serjeant et al. 2002):

$\left[\frac{\operatorname{SFR}\left(M \geq 0.1 M_{\odot}\right)}{M_{\odot} \mathrm{yr}^{-1}}\right]=1.1 \times 10^{-21}\left(\frac{L_{v}}{\mathrm{~W} / \mathrm{Hz}}\right)\left(\frac{v}{\mathrm{GHz}}\right)^{-\alpha}$

where $L_{v}$ is the non-thermal luminosity at the frequency $v$ (in our case $1.344 \mathrm{GHz}$ ), and $\alpha$ is the non-thermal spectral index. The SFR of the 11 emission line galaxies detected in A3921 central field are summarised in Table 5.

We also estimated the SFR of the star-forming galaxies in A3921 from their [OII] emission, adopting the relation by Kennicutt (1998) ${ }^{3}$ :

$$
\begin{aligned}
{\left[\frac{\operatorname{SFR}\left(M \geq 0.1 M_{\odot}\right)}{M_{\odot} \mathrm{yr}^{-1}}\right] } & =1.4 \times 10^{-34}\left(\frac{L_{[\mathrm{OII}]}}{\mathrm{W}}\right) \\
& \simeq 2.0 \times 10^{-12} \frac{L_{B}}{L_{B_{\odot}}} E W([\mathrm{OII}]) E\left(\mathrm{H}_{\alpha}\right)
\end{aligned}
$$

where we used the relation by Kennicutt (1992) to determine the luminosity of the [OII] emission line from a) its equivalent widths $(E W([\mathrm{OII}]))$, b) the integrated broad-band $B$ luminosity of the galaxy in solar units $\left(L_{B} / L_{B_{\odot}}\right)$, and c) a reasonable value for the extinction correction $\left(E\left(\mathrm{H}_{\alpha}\right)\right)$. In Table 5 we give the SFR from $E W([\mathrm{OII}])$ both with and without a correction for dust-extinction. When included, a canonical value for extinction ( 1 mag for $\mathrm{H}_{\alpha}$ ) has been applied. In Eq. (5) the errors can be considerably large $( \pm 30-50 \%)$, mostly due to the adopted relation between the $B$-band and [OII] line luminosities (Kennicutt 1992).

The results are summarised in the upper part of Table 5, which lists:

- Cols. (1), (2): optical and radio ID (corresponding to Table A in F05 and Table 3 in this paper, respectively);

- Cols. (3), (4): optical coordinates;

- Cols. (5), (6): redshift and spectral type of the source;

- Col. (7): Log of the absolute spectral luminosity at $1.344 \mathrm{GHz}(\mathrm{W} / \mathrm{Hz})$. For the sources not detected at radio wavelengths, we estimated an upper limit (at $3 \sigma$ significance level) using $S_{1.344 \mathrm{GHz}}<0.147 \mathrm{mJy} /$ beam and $\alpha=-0.8$;

- Cols. (8), (9): SFR of the source estimated from its [OII] line luminosities, without $\left(E\left(\mathrm{H}_{\alpha}\right)=0\right)$ and with $\left(E\left(\mathrm{H}_{\alpha}\right)=1\right)$ the extinction correction;

- Col. (10): SFR estimated from the $1.344 \mathrm{GHz}$ luminosity of the source.

These results are discussed in the following section.

\subsubsection{Emission line galaxies with detected radio emission}

Among the 11 emission-line cluster members detected in A3921 by F05, only two have detectable radio emission at our flux limit $(0.15 \mathrm{mJy} /$ beam, $3 \sigma$ level $)$.

One (R2) is very likely a classical spiral galaxy, as suggested by most of its observed properties. Firstly, based on the presence and strength of [OII] emission and $\mathrm{H}_{\delta}$ absorption lines, F05 classified R2 as an e(c)-type object (MORPHS classification; Dressler et al. 1999). Following Poggianti et al. (1999), e(c) spectra are typical for present-day spirals. This conclusion agrees with the optical morphology and the radio SFR of R2,

\footnotetext{
3 SFRs from $E W([\mathrm{OII}])$ had already been estimated in F05. Here we re-estimated these SFRs with the same IMF adopted in the previous radio relation.
} 
Table 4. Optical identification of the $>5 \sigma$ radio sources. "-" indicates unknown values. R5 and R3 correspond to BG2 and BG3 respectively in F05. ${ }^{*}$ Indicates uncertain $z$ (Q.F. $>1$ in F05). ${ }^{* *} z$ from ENACS catalogue, not from F05.

\begin{tabular}{|c|c|c|c|c|c|c|c|c|c|c|}
\hline$\overline{\mathrm{IID}}$ & $\overline{\mathrm{II}} \mathrm{ID}_{\mathrm{O}}$ & $\begin{array}{c}\mathrm{RA}_{\mathrm{J} 2000} \\
\mathrm{~h} \mathrm{~m} \mathrm{~s}\end{array}$ & 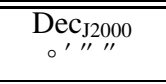 & $\overline{\overline{\Delta_{\mathrm{r}-\mathrm{o}}}}$ & $\overline{\mathcal{R}}$ & $\begin{array}{c}V \\
\text { mag }\end{array}$ & $\begin{array}{c}R \\
\mathrm{mag}\end{array}$ & $\begin{array}{c}I \\
\mathrm{mag}\end{array}$ & $\bar{z}$ & $\begin{array}{c}\log L_{22 \mathrm{~cm}} \\
\left(h_{75}{ }^{-2} \mathrm{WHz}^{-1}\right)\end{array}$ \\
\hline R1 & - & 224835.32 & -642038.2 & 0.98 & 1.43 & 22.48 & 22.04 & - & - & - \\
\hline R2 & 57 & 224839.75 & -641922.2 & 0.46 & 0.21 & 17.01 & 16.44 & 15.79 & 0.091 & 21.82 \\
\hline R3 & 69 & 224849.01 & -642311.2 & 1.75 & 1.65 & 16.22 & 15.59 & 14.84 & 0.097 & 22.59 \\
\hline R4 & 215 & 224902.64 & -642743.6 & 0.41 & 0.51 & 19.60 & 19.45 & 19.41 & $0.065^{*}$ & 21.56 \\
\hline R5 & 52 & 224904.78 & -642035.6 & 1.17 & 1.71 & 15.42 & 14.72 & 14.00 & 0.093 & 24.00 \\
\hline R6 & _** & 224904.93 & -641816.2 & 0.37 & 0.45 & 17.21 & 16.63 & 15.93 & $0.136^{*}$ & 22.93 \\
\hline R9 & 39 & 225006.44 & -642442.4 & 8.43 & 12.40 & 16.38 & 15.73 & 14.99 & 0.094 & 24.12 \\
\hline $\mathrm{R} 10$ & - & 225013.59 & -641936.1 & 0.84 & 0.69 & 17.64 & 17.18 & 16.65 & - & - \\
\hline R11 & - & 225026.50 & -642820.1 & 0.69 & 0.86 & 17.69 & 17.24 & 16.67 & - & - \\
\hline R12 & - & 225035.40 & -642546.8 & 4.24 & 1.64 & 20.56 & 19.21 & 18.22 & - & - \\
\hline R13 & - & 225036.62 & -641607.6 & 1.05 & 0.58 & - & 20.57 & 19.56 & - & - \\
\hline R14 & _** & 225038.22 & -642021.1 & 1.00 & 0.49 & 16.73 & 16.33 & 15.88 & $0.093^{*}$ & 22.20 \\
\hline R15 & - & 225046.78 & -641700.9 & 1.88 & 2.77 & - & 23.22 & - & - & - \\
\hline R16 & - & 225050.62 & -64 2719.8 & 0.29 & 0.31 & - & 22.92 & 21.35 & - & - \\
\hline
\end{tabular}

typical of late-type objects. The red colour of this galaxy and the low $\mathrm{SFR}_{[\mathrm{OII}]}$ (significantly lower than the radio estimate) could be due to the fact that, in the optical, the younger (disk) component of the galaxy is much more obscured by dust lanes than the old bulge.

The other emission-line galaxy detected at radio wavelengths (R3) is associated with the third brightest cluster galaxy (BG3 in F05), and it is located at the barycentre of the X-ray emission of A3921-B (B05). In optical it is characterised by: a) an elliptical morphology; b) red $(V-R)_{A B}$ and $(R-I)_{A B}$ colours; c) a high radial velocity offset from the mean cluster redshift $\left(+788 \mathrm{~km} \mathrm{~s}^{-1}\right)$; d) strong [OII] emission $(E W([\mathrm{OII}])=-65 \AA)$. Based on its spectral properties BG3 was classified as a star-burst galaxy by F05, but, based on its nebular emission line ([OII]), it could also be a Seyfert or a LINER. Unfortunately, the very limited spectral range covered by the optical observations did not allow F05 to use any diagnostic diagrams to distinguish between these two hypotheses.

We therefore analysed in detail the radio and X-ray (B05) properties of BG3. Following Morrison \& Owen (2003) and correcting for our cosmology, we considered as AGNs the radio galaxies with $\log \left(L_{1.4 \mathrm{GHz}}(\mathrm{W} / \mathrm{Hz})\right) \gtrsim 22.73$, and as starforming objects those below this limit. The radio luminosity of BG3 $\left(\log \left(L_{22} \mathrm{~cm}(\mathrm{~W} / \mathrm{Hz})\right)=22.59\right)$ is on the upper side of the luminosity distribution of star-forming galaxies, albeit very close to the boundary with AGNs. Thus, from a statistical point of view, BG3 has radio properties typical of starforming/bursting objects, but we cannot completely exclude the faint AGN hypothesis.

The XMM analysis of A3921 by B05 revealed a diffuse extended X-ray emission for BG3, with a thermal spectrum. This points against an AGN origin of the emission, and we suggest that the intense star-burst episode observed in BG3 has probably injected hot gas into the galaxy's inter-stellar medium (ISM). Both the red colours and the strong difference between the optical and radio SFR estimates (see Table 5) could be due to dust extinction. Actually, it has been shown that, in clusters, star-forming galaxies heavily affected by dust extinction are more centrally concentrated than star-forming galaxies in general (Miller \& Owen 2002). MIR observations also revealed a higher fraction of dusty-star-burst galaxies in dynamically young clusters (Coia et al. 2005), as in the case of A3921. Therefore, we concluded that the red colours of R2 and R3 are probably due to their dusty star-forming/bursting nature (e.g. Wolf et al. 2005).

\subsubsection{Emission line galaxies without detected radio emission}

None of the other nine emission line cluster members were detected at the $3 \sigma$ sensitivity limit of our radio observations. These galaxies are thus characterised by SFRs lower than 3-4 $M_{\odot} \mathrm{yr}^{-1}$.

We suggest that the SF properties of the emission-line galaxies detected in the collision region of A3921 may be due to cluster environmental effects. Most of them are brighter than the "dwarfs/giants" magnitude cut applied by Poggianti et al. (2004) in their analysis of the Coma cluster (see F05), and they all have SFRs lower than those typical of gas-rich spirals $\left(\sim 20 M_{\odot} \mathrm{yr}^{-1}\right.$, e.g. Kennicutt 1998). They could be gas-poor objects, whose gas deficiency is related to cluster environmental effects, and in particular to hydrodynamical interactions between the galaxy ISM and the ICM (ram-pressure and viscous stripping, thermal evaporation; see Boselli \& Gavazzi 2006 for a recent and complete review).

The sub-cluster merger in A3921 could have subsequently enhanced the SF activity of the galaxies located in the collision region. It has been shown that both dynamical interactions with the ICM and tidal interactions with nearby companions and/or the cluster potential can induce SF in cluster galaxies (e.g. Gavazzi et al. 1995; Moss \& Whittle 2000; Bekki 1999). Actually, these physical mechanisms are particularly effective in merging clusters since: a) the ISM compression due to galaxyICM interactions is most efficient where the density and the temperature of the ICM reach their maximum (i.e. around the ICM compression bar in A3921 detected by B05), and b) the timevarying gravitational potential of merging clusters enhances the rate of galaxy encounters and drives efficient transfer of gas to the central region of galaxies, triggering a starburst.

We can extract more physical information about some of the emission-line galaxies without radio emission at the $3 \sigma$ level by comparing in more detail their optical and radio properties. One of these objects has spectral features typical of star-bursting galaxies $\left(\mathrm{ID}_{\mathrm{O}}=100\right.$ in Table 5), i.e. strong [OII] emission ( $E W<-40 \AA$ ). Its distorted optical morphology (Fig. 4) suggests that the observed star-burst episode could be due to interaction with another galaxy.

Three galaxies have spectra typical of spirals forming stars at a constant rate $(\mathrm{e}(\mathrm{c}))$, while the remaining 5 belong to the 

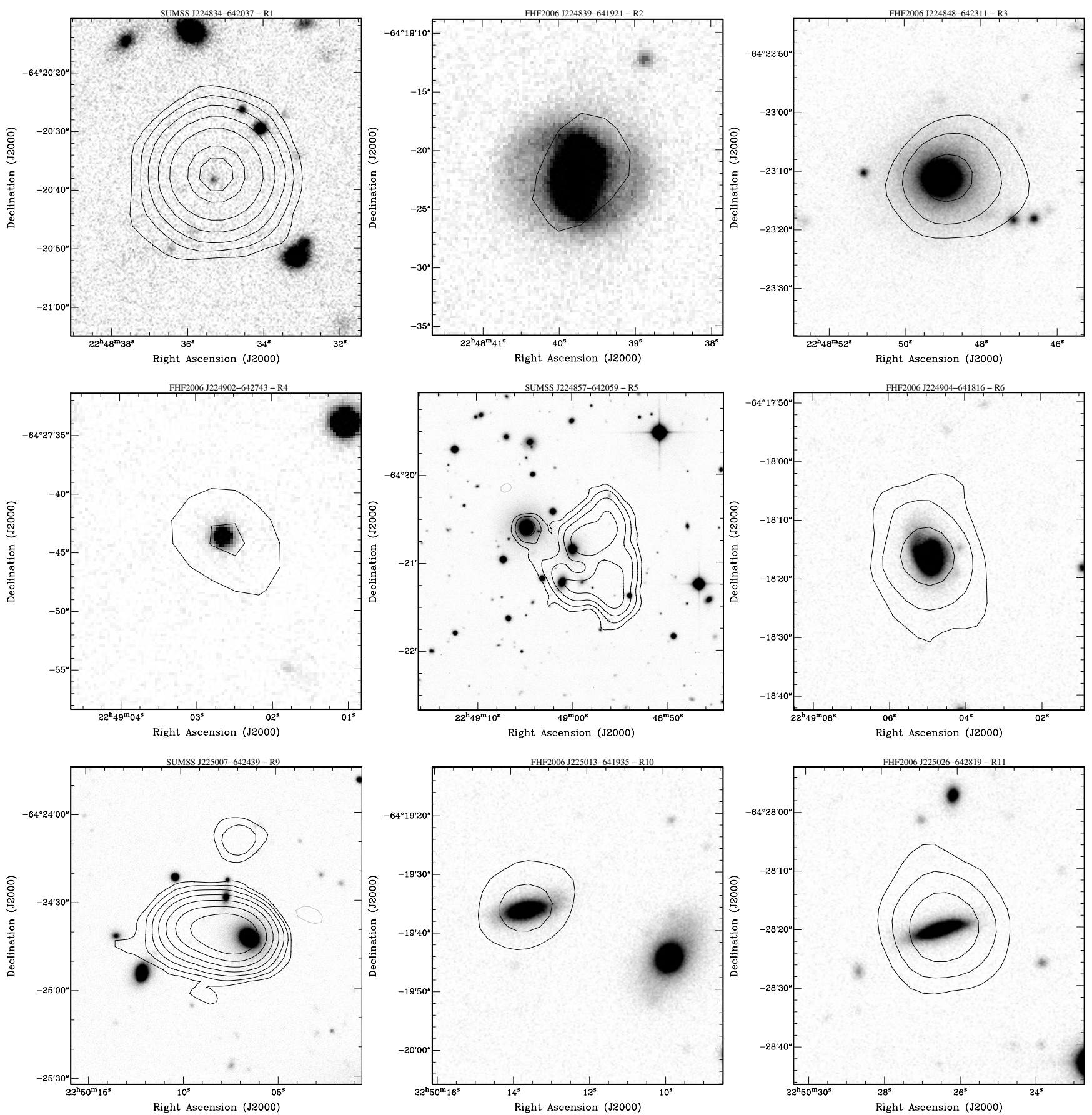

Fig. 3. $1.344 \mathrm{GHz}$ radio contours of the radio sources in the A3921 central field overlaid on the corresponding $R$-band image. When not specified, radio contours are as follow: $0.245 \mathrm{mJy} /$ beam $\times(-1,1,2,4,8,16,32,64, \ldots)$. For R1 (SUMSS J224834-642037) radio contours are: $0.245 \mathrm{mJy} / \mathrm{beam} \times(-1,1,2,4,8,15,30,40)$; for R4 (FHF2006 J224902-642743): $0.245 \mathrm{mJy} / \mathrm{beam} \times(-1,1,1.5)$. R3 (FHF2006 J224848-642311) and R5 (SUMSS J224857-642059) are associated with the third and the second brightest cluster galaxies (BG3 and BG2 in F05).

e(a) class, that, based on spectral modelling (Poggianti et al. 1999), could be either dusty star-burst or post-star-burst galaxies with some residual SF. Up to now only e(a) galaxies belonging to the first class have been detected, showing extremely strong dust extinction factors (median value $\sim 12$ mag, Duc et al. 2002). For our e(a) objects, by dividing the 8 th and 10th columns of Table 5, we find $S F R_{1.4 \mathrm{GHz}} / S F R_{[\mathrm{OII}]}<13-79$, corresponding to dust extinction values $<5-30 \mathrm{mag}$ (lower than 12 mag for $4 / 5$ of the sample). This means that, for extinction higher than these lower limits, we should observe the e(a) galaxies if they were dusty star-burst. Since the estimated extinction factors are comparable to those found by Duc et al. (2002) and we detected none of the e(a) galaxies at radio wavelengths, we suggest that at least a fraction of them could not be dusty star-burst, but poststar-burst galaxies with some residual SF. This hypothesis has to be tested with more sensitive observations (e.g. higher-resolution spectroscopy covering a wider wavelength range, IR and deeper radio observations) as our SFR determination has big errors. The highest uncertainty is related to the SFR derived from the [OII] line, which suffers from several problems other than extinction (e.g. slit aperture and metallicity effects). 

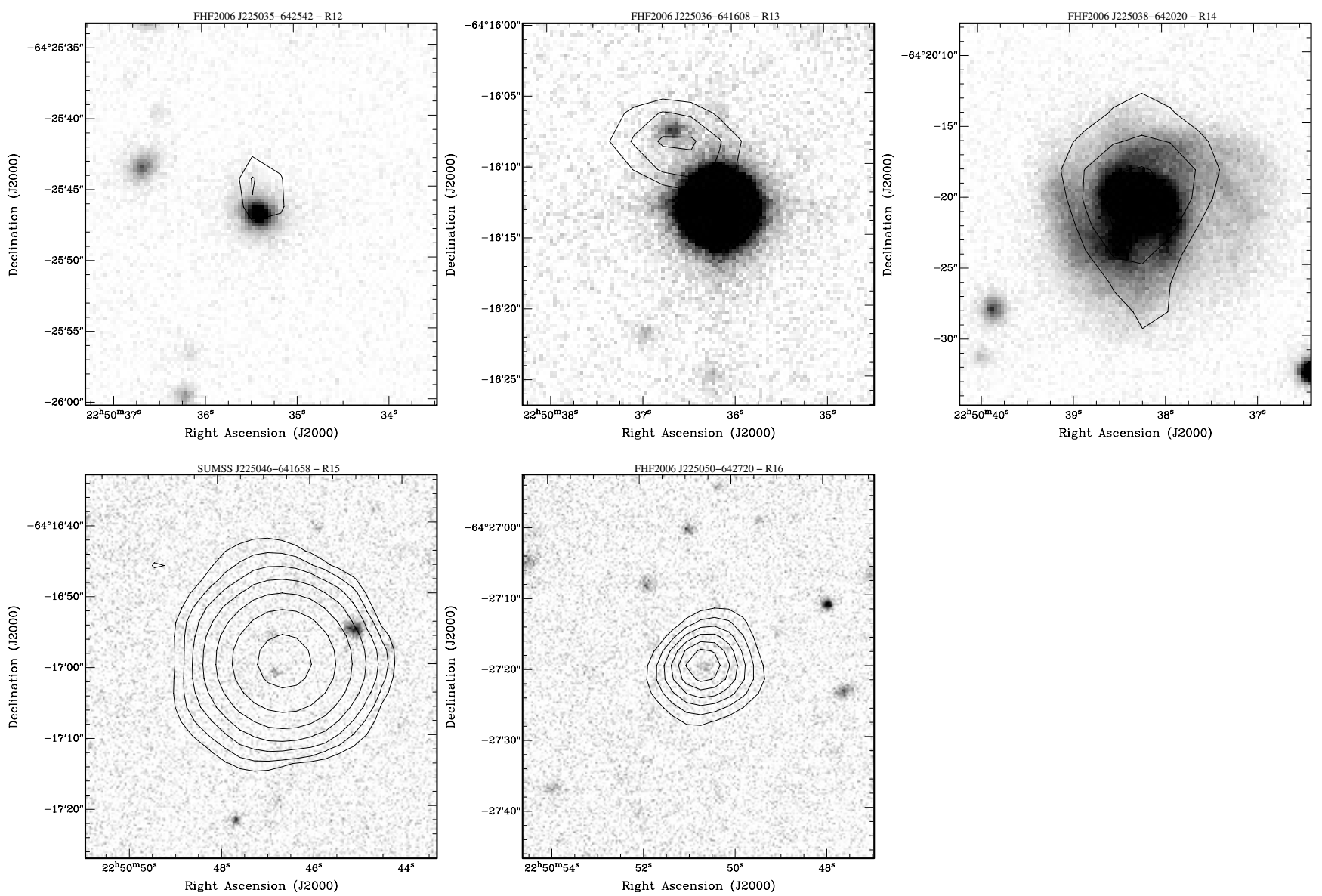

Fig. 3. continued. For R12 (FHF2006 J225035-642542) radio contours are: $0.2205 \mathrm{mJy} /$ beam $\times(-1,1,1.1)$ (i.e. \pm 4.5 and $5 \sigma$ level); for R13 (FHF2006 J225036-641608) and R14 (FHF2006 J225038-642020): 0.245 mJy/beam × (-1, 1, 1.2, 1.4); for R16 (FHF2006 J225050-642720): $0.245 \mathrm{mJy} /$ beam $\times(-1,1,1.4,1.8,2.2,2.6,3)$.

Table 5. Cluster members with emission lines (and possible radio emission) (top), and with radio emission but no emission lines (bottom). The optical ID corresponds to Table A in F05. The coordinates are those of the optical position. Top: the SFR of the emission line cluster members was measured through their [OII] $\left({ }^{\diamond}\right.$ : extinction correction term $E\left(\mathrm{H}_{\alpha}\right)=0{ }^{\diamond}{ }^{\diamond}$ : extinction correction term $\left.E\left(\mathrm{H}_{\alpha}\right)=1.\right)$ and $1.4 \mathrm{GHz}$ luminosities. In case of no radio detection an upper limit $(3 \sigma)$ was estimated. Bottom: confirmed and candidate cluster members detected at radio wavelengths and either without optical emission lines or unknown spectral type. Among the objects with available $z$, spectral types are available neither for poor S/N (Q.F. > 1 in F05) nor for ENACS (Katgert et al. 1996, 1998; Mazure et al. 1996) spectra. For candidate cluster members we assumed the mean cluster redshift. *: Object from the ENACS catalogue.

\begin{tabular}{|c|c|c|c|c|c|c|c|c|c|}
\hline $\mathrm{ID}_{\mathrm{O}}$ & $\mathrm{ID}_{\mathrm{R}}$ & $\begin{array}{c}\mathrm{RA} \\
(\mathrm{J} 2000) \\
\mathrm{h} \mathrm{m} \mathrm{s}\end{array}$ & $\begin{array}{c}\text { Dec } \\
(\mathrm{J} 2000) \\
\circ^{\prime} \prime \prime\end{array}$ & $z$ & Sp. type & $\begin{array}{c}\log L_{22 \mathrm{~cm}} \\
\left(h_{75}{ }^{-2} \times\right. \\
\mathrm{W} / \mathrm{Hz}) \\
\end{array}$ & $\begin{array}{c}S F R_{[\mathrm{OII}]}{ }^{\diamond} \\
\left(h_{75}{ }^{-2} \times\right. \\
\left.M_{\odot} \mathrm{yr}^{-1}\right)\end{array}$ & $\begin{array}{c}S F R_{[\mathrm{OII}]} \diamond \\
\left(h_{75}{ }^{-2} \times\right. \\
\left.M_{\odot} \mathrm{yr}^{-1}\right) \\
\end{array}$ & $\begin{array}{c}S F R_{1.4 \mathrm{GHz}} \\
\left(h_{75}{ }^{-2} \times\right. \\
\left.M_{\odot} \mathrm{yr}^{-1}\right)\end{array}$ \\
\hline 17 & - & 224859.0 & -642111.8 & 0.094 & $\overline{e(a)}$ & $<21.42$ & 0.28 & 0.70 & $<3.68$ \\
\hline 45 & - & 224941.5 & -642624.1 & 0.085 & $e(c)$ & $<21.33$ & 0.04 & 0.11 & $<2.98$ \\
\hline 57 & $\mathrm{R} 2$ & 224839.8 & -641922.2 & 0.091 & $e(c)$ & 21.82 & 0.25 & 0.62 & 7.94 \\
\hline 69 & R3 & 224849.0 & -642311.2 & 0.097 & $e(b)$ & 22.59 & 5.64 & 14.09 & 50.35 \\
\hline 73 & - & 224834.8 & -642339.9 & 0.095 & $e(c)$ & $<21.43$ & 0.26 & 0.64 & $<3.76$ \\
\hline 81 & - & 224941.1 & -642405.6 & 0.097 & e(a) & $<21.45$ & 0.05 & 0.12 & $<3.93$ \\
\hline 82 & - & 224938.4 & -642323.8 & 0.099 & e(a) & $<21.47$ & 0.16 & 0.40 & $<4.11$ \\
\hline 100 & - & 224941.6 & -641959.0 & 0.086 & e(b) & $<21.34$ & 0.70 & 1.74 & $<3.05$ \\
\hline 169 & - & 225001.8 & -642221.8 & 0.085 & e(a) & $<21.33$ & 0.12 & 0.31 & $<2.98$ \\
\hline 181 & - & 224926.1 & -642321.4 & 0.095 & $e(c)$ & $<21.43$ & 0.10 & 0.24 & $<3.76$ \\
\hline 226 & - & 224912.0 & -641603.9 & 0.100 & e(a) & $<21.48$ & 0.17 & 0.43 & $<4.20$ \\
\hline 39 & R9 & 225006.4 & -642442.4 & 0.094 & $\mathrm{k}$ & 24.12 & & & \\
\hline 52 & R5 & 224904.8 & -642035.6 & 0.093 & $\mathrm{k}$ & 24.00 & & & \\
\hline$-{ }^{*}$ & $\mathrm{R} 14$ & 225038.2 & -642021.1 & 0.093 & - & 22.20 & & & \\
\hline- & $\mathrm{R} 10$ & 225013.6 & -641936.1 & - & - & 22.26 & & & \\
\hline- & $\mathrm{R} 11$ & 225026.5 & -642820.1 & - & - & 22.67 & & & \\
\hline
\end{tabular}




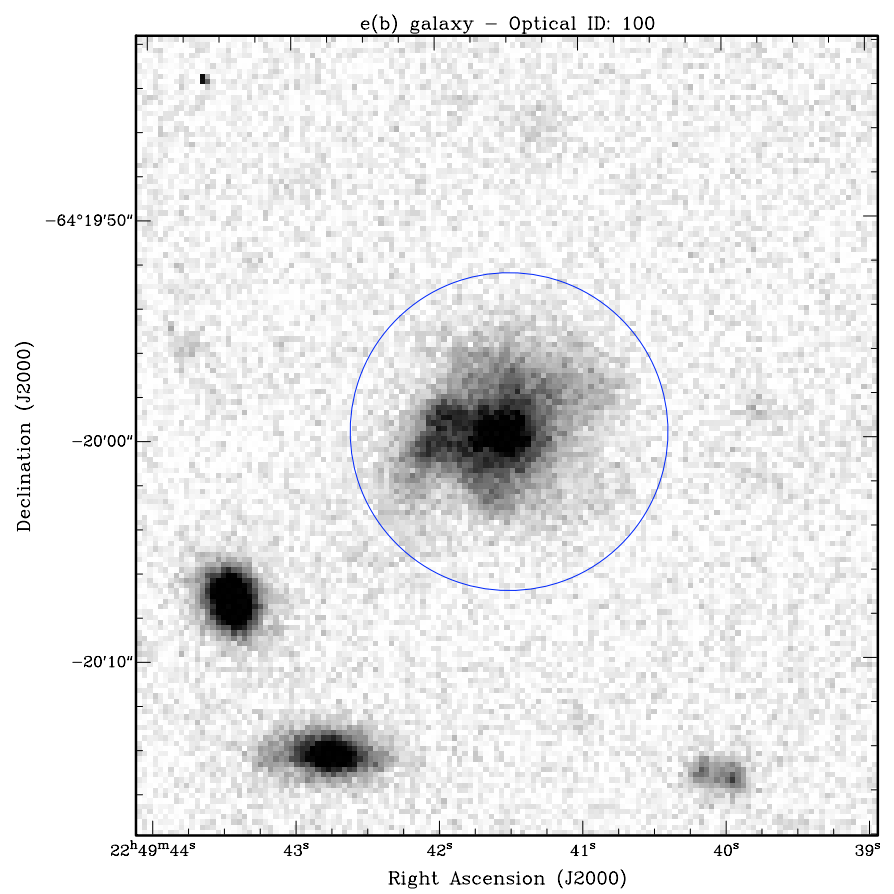

Fig. 4. $R$-band image of the $\mathrm{e}(\mathrm{b})$ galaxy with optical $\mathrm{ID}=100$ in Table 5 .

\subsection{4. $\mathrm{k}+\mathrm{a}$ galaxies}

Among the cluster members observed by F05, 13 are characterised by an intermediate or strong $\mathrm{H}_{\delta}$ line in absorption and no detectable [OII] 3727 line in emission $(\mathrm{k}+$ a galaxies; Dressler et al. 1999). These galaxies are generally interpreted as objects that have no significant ongoing SF, but that were forming stars in the recent past $(\lesssim 1-1.5 \mathrm{Gyr})$. They are therefore usually classified as post-star-forming (moderate Balmer lines, $E W\left(\mathrm{H}_{\delta}\right)<4-5 \AA$ ) or post-star-bursting objects (strong Balmer lines, $\left.E W\left(\mathrm{H}_{\delta}\right)>4-5 \AA\right)$. However, it has been suggested that $\mathrm{k}+\mathrm{a}$ (like e(a)) might also be star-bursting galaxies whose emission lines are invisible in the optical due to heavy or selective dust extinction (e.g. Smail et al. 1999; Poggianti \& Wu 2000; Goto 2004). Understanding the SF properties of the $\mathrm{k}+\mathrm{a}$ galaxies in A3921 is important firstly to address the debated nature of this class of objects, and secondly to confirm (or reject) the spatial correlation between the detected star-forming objects and the collision region of the cluster.

None of our target $\mathrm{k}+\mathrm{a}$ galaxies was detected down to our $2 \sigma$ sensitivity limit $\left(S F R \sim 3 M_{\odot} \mathrm{yr}^{-1}\right.$ at the mean redshift of the cluster and assuming $\alpha=-0.8$ ). This means that no galaxies of this spectral class host dust obscured SF activity up to our $2 \sigma$ sensitivity limit $\left(\sim 3 M_{\odot} \mathrm{yr}^{-1}\right)$. Based also on the moderate $\mathrm{H}_{\delta}$ equivalent width and on the red colours of the $\mathrm{k}+\mathrm{a}$ galaxies detected in A3921 (F05), we can therefore conclude that they are not strong dusty star-burst, and, in agreement with the results found by Duc et al. (2002) in the cluster A1689, we suggest that these objects are in a post-star-forming phase as predicted by their spectral properties. Due to the limit in sensitivity of our radio observations, we cannot, however, totally exclude the possibility that they are dust-obscured galaxies forming stars at a very low rate. The biggest issue in this respect is that the spectroscopic classification of these objects is based solely on the $[\mathrm{OII}]$ emission and Balmer absorption lines. The importance of having information as well on the $\mathrm{H}_{\alpha}$ line has been shown (Goto 2004). An ongoing spectroscopic follow-up covering a wider spectral range will allow us to definitively test the poststar-forming nature of the $\mathrm{k}+\mathrm{a}$ galaxies in A3921.

\subsection{Radio galaxies}

In the bottom panel of Table 5 we list the five confirmed or candidate cluster members i) with detected radio emission, and ii) without emission lines, or for which the spectral type is unknown. Three of them (R10, R11 and R14) are located in the Eastern region of A3921-A (i.e. not in the collision region), and they are very likely gas rich, dusty star-forming late-type galaxies, since they have: i) radio luminosities below the AGN/SF boundary $\left(\log \left(L_{1.4 \mathrm{GHz}}(\mathrm{W} / \mathrm{Hz})\right)=22.73\right.$, see Sect. 4.1.2); ii) spiral-like morphologies (R14 also shows a disturbed morphology, suggesting that the SF activity could be triggered by galaxygalaxy interaction); and iii) red colours probably due to dust extinction. The remaining two radio emitting cluster members (R9 and R5) are on the contrary AGNs, their optical morphology and spectrum being typical of early-type galaxies and their radio luminosity being well above the AGN/SF boundary. We therefore detected only two AGNs in the central field of A3921. A similar number of AGNs was detected in the nearby clusters Coma and A1367 (Gavazzi \& Boselli 1999). In the central $\sim 1.8 \times 1.5 \mathrm{Mpc}^{2}$ region of these two systems, 3 and 2 galaxies respectively have radio luminosities above the adopted AGN/SF boundary (from Table 1 of Gavazzi \& Boselli 1999).

The small number of radio galaxies did not allow us to determine the radio luminosity function (RLF) for early-type galaxies. We compared the number of detected radio galaxies with the result of Ledlow \& Owen (1996), who detected $12 \%$ of galaxies with optical magnitude $R \leq 17.5$ and radio luminosity $\log \left(L_{1.4 \mathrm{GHz}}(\mathrm{W} / \mathrm{Hz})\right)>22.02$ (scaled to our cosmological parameters). In the same radio and optical range, we found six cluster members, of which three have clear elliptical morphology, and two can be safely classified as AGN. Due to the lack of complete spectroscopy, we assumed that all the red sequence galaxies with $R \leq 17.5$ belong to A3921, finding in total 36 galaxies. Therefore, the fraction of radio emitting elliptical galaxies in our sample is $8 \%$, and the fraction of AGN is $6 \%$. Taking into account the uncertainty on the total number of galaxies and the fact that these percentages are derived from very small numbers, we conclude that our result is not significantly different from that of Ledlow \& Owen (1996).

\section{The peculiar radio source SUMSS J224857-642059 (R5)}

\subsection{Total intensity and spectral index images}

The most interesting radio emission observed in the central field of A3921 is the extended source SUMSS J224857-642059 (R5) (see Fig. 5). In Fig. 6, we have superimposed the $1.344 \mathrm{GHz}$ radio contours on the $R$-band optical image of A3921 from F05. The radio component in the North-East extremity of R5 is coincident with a very bright galaxy $\left(R_{A B}=16.1\right)$ corresponding to the second brightest cluster member ("BG2" in F05), whose colour and spectrum are typical of an early-type object. BG2 is the dominant galaxy of one of the two colliding sub-clusters revealed by the combined optical/X-ray analyses of the cluster by F05 and B05.

The most likely hypothesis is that R5 is a narrow-angle tail (NAT) radio galaxy, whose core component is associated with the galaxy BG2. A source with very similar structure has been detected by Gregorini \& Bondi (1989) in A115. The jets in R5 


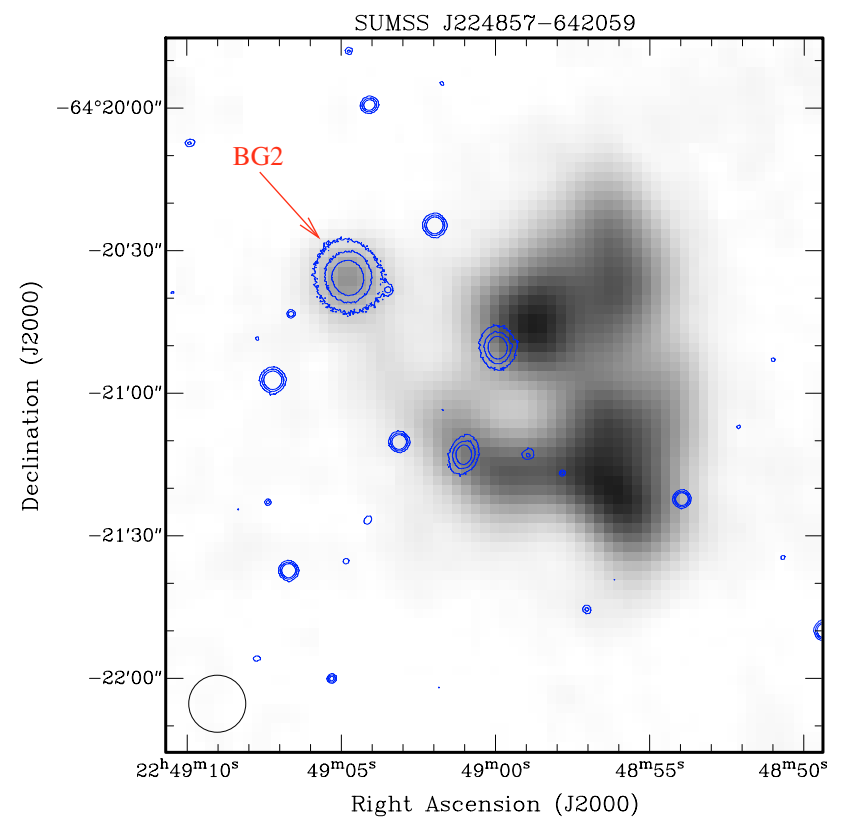

Fig. 5. $R$-band contours overlaid on the $1.344 \mathrm{GHz}$ image of the peculiar radio source SUMSS J224857-642059 (R5). The beam size (shown in the bottom-left corner) is $12^{\prime \prime} \times 12^{\prime \prime}$.

are not visible at our resolution, and the tails of diffuse emission, embedded in a common low-brightness envelope, are oriented along a NE/SW (d1) and a SE/NW (d2) axis (Fig. 8). The main elongation axis of the tail (d1) is roughly aligned with the direction along which A3921-B is tangentially traversing A3921-A (SW/NE, see Fig. 7). This agrees with the accepted view that NAT morphologies are the result of the ram-pressure exerted by the ICM on the radio plasma, the ram-pressure being due to relative motion between the ICM and the radio emitting galaxy (Blandford \& Rees 1974). The radial velocity offset of BG2 from the mean radial velocity of the cluster is $-145 \pm 79 \mathrm{~km} \mathrm{~s}^{-1}$. However, the proper velocity of BG2 with respect to the ICM could be higher, since it has been shown that the merging axis of A3921-B is nearly on the plane of the sky (F05), resulting in a small velocity component along the line of sight.

We obtained the spectral index map of the radio source R5 by combining the images at $1.344 \mathrm{GHz}$ and $2.368 \mathrm{GHz}$ with the AIPS task COMB (Fig. 8). We note a steepening of the spectrum going from the region around BG2 to the extended emission, supporting the classification of R5 as a tailed radio galaxy. In particular, the core component coincident with BG2 shows a significantly flat spectral index $(\langle\alpha\rangle=-0.4)$, with a steepening of the spectrum to $\alpha=-1 \div-1.5$ on the SW side.

The trend of the spectral index of the R5 tails along the main axes (d1 and d2 in Fig. 8) is shown in Fig. 9. The tail spectrum is very steep in both directions. We found a steepening spectral index going away from BG2 along $\mathrm{d} 2$, while the spectrum shows more jumps along $\mathrm{d} 1$.

The physical properties of the extended radio source $\mathrm{R} 5$ are summarised in Table 6. Equipartition magnetic field was calculated with the standard assumptions of equal energy density in protons and electrons, a magnetic field filling factor of 1 , and a spectrum extending from $10 \mathrm{MHz}$ to $100 \mathrm{GHz}$. The spectral index was taken $\alpha=-1.5$ and the source depth along the line of sight of $50 h_{75}{ }^{-1} \mathrm{kpc}$. The source age given in the table refers to lifetime of electrons radiating at $1.4 \mathrm{GHz}$ in a magnetic field taken at the equipartition value.

\subsection{Polarization images}

Images in the Stokes parameters $U$ and $Q$ were produced analogously to the I maps of the cluster by INVERTing, CLEANing and RESTORing the data with the MIRIAD software. In particular, for each pointing, the $(u, v)$ data at the same frequency of all the different configurations were handled together. We restored the $2.368 \mathrm{GHz}$ maps with a beam size of both $8^{\prime \prime} \times 8^{\prime \prime}$ (i.e. $13 \mathrm{~cm}$ beam size), and $12^{\prime \prime} \times 12^{\prime \prime}$ (i.e. $22 \mathrm{~cm}$ beam size). The images of each field were then mosaiced using the task LINMOS.

Using the MIRIAD task IMPOL, we derived from the I, Q and $\mathrm{U}$ maps the images of: a) the polarised intensity $P=\left(Q^{2}+\right.$ $\left.U^{2}\right)^{1 / 2}$, b) the degree of polarization $\left.m=P / I, \mathrm{c}\right)$ the position angle of polarization (defined to be positive North through East) $\psi=0.5 \tan ^{-1}(U / Q)$, and $\left.\mathrm{d}\right)$ the associated errors. These maps were all blanked when the error in the position angle image was greater than $10^{\circ}$ or when the $S / N$ ratio in the polarised intensity map was less than three.

The source R5 is polarized in the Northern region both at 1.344 and $2.368 \mathrm{GHz}$. In the radio images presented in Fig. 6 the vectors indicate the orientation of the projected $\mathbf{E}$-field and are proportional in length to the fractional polarization. The mean fractional polarization is $\sim 11 \%$ and $\sim 40 \%$ respectively at 1.344 and $2.368 \mathrm{GHz}$, reaching values of $\sim 34 \%$ and $\sim 60 \%$ at the Northern boundary of the source, whereas for the Southern tail we can set upper limits of $\sim 6 \%$ and $\sim 20 \%$ at 1.344 and 2.368 $\mathrm{GHz}$ respectively. A possible interpretation of the different polarization percentage in the two tails is that the source is strongly affected by projection effects.

As the polarized emission from a radio source passes through a magnetized, ionized plasma, its plane of polarization will be rotated by the Faraday effect:

$\psi_{\lambda}=\psi_{0}+R M \lambda^{2}$

where $\psi_{\lambda}$ and $\psi_{0}$ are the observed and intrinsic polarization angles respectively and $\lambda$ is the wavelength at which observations are performed. $R M$ is the Rotation Measure, which depends on the product of the electron density $\left(n_{\mathrm{e}}\right)$ and magnetic field along the line of sight $\left(H_{\|}\right)$. Due to this effect, the plane of polarized radiation from cluster and background radio galaxies may be rotated if magnetic fields are present in the ICM.

We derived the rotation measure $(R M)$ and the zerowavelength position angle $\left(\psi_{0}\right)$ maps by fitting Eq. (6) to the position angle images of the radio galaxy R5 at $1.344 \mathrm{GHz}$ and $2.368 \mathrm{GHz}$ with a resolution of $12.0^{\prime \prime}$. For this, we used the MIRIAD task IMRM. The measured RM values range from 11.2 to $33.5 \mathrm{rad} / \mathrm{m}^{2}$. They are consistent with the Galactic contribution, which in the region of A3921 is expected to be about $30 \mathrm{rad} / \mathrm{m}^{2}$ (Simard-Normandin et al. 1981). This suggests that the cluster magnetic field in this region may be low or mostly perpendicular to the line of sight. The orientation of the magnetic field of R5 is roughly longitudinal as commonly found in tailed radio sources (Dallacasa et al. 1989; Feretti et al. 1998).

\section{Discussion and conclusions}

\subsection{Star-forming galaxies and AGNs: possible connections with the ongoing merger}

In this paper we presented the 22 and $13 \mathrm{~cm}$ ATCA observations of the galaxy cluster A3921. We concentrated our analysis on the central field of the cluster $\left(0.075 \mathrm{deg}^{2}\right)$, which hosts two merging sub-clusters (A3921-A and A3921-B) that have not yet undergone the first core-core encounter (B05; F05; 

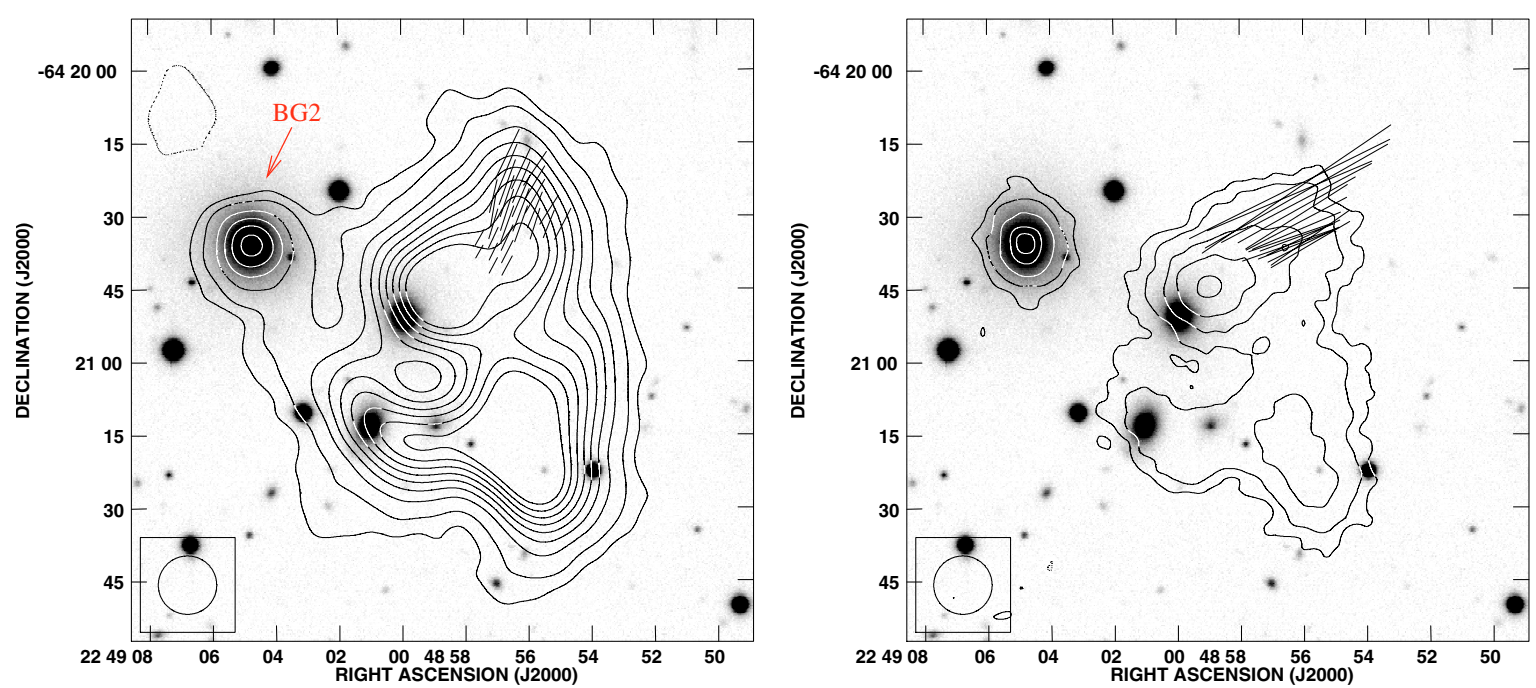

Fig. 6. $1.344 \mathrm{GHz}$ (left) and $2.368 \mathrm{GHz}$ (right) radio contours of the extended radio source SUMSS J224857-642059 (R5), superimposed on the $R$-band optical image (WFI observations, F05). The radio emission is associated with the second brightest cluster galaxy (BG2 in F05). In both images the angular resolution is $12^{\prime \prime} \times 12^{\prime \prime}$. Left: the contour levels are: $0.147 \mathrm{mJy} /$ beam $\times(-1,1,2,4,6,8,10,12,14,16)$. The r.m.s. noise level of the map is $0.049 \mathrm{mJy} / \mathrm{beam}$. Right: the contour levels are: $0.159 \mathrm{mJy} /$ beam $\times(-1,1,2,4,6,8,10,12,14,16)$. The r.m.s. noise level of the map is $0.053 \mathrm{mJy} / \mathrm{beam}$. In both panels, superimposed lines represent the orientation of the electric vector $(\boldsymbol{E})$ and are proportional in length to the fractional polarization $\left(1^{\prime \prime}=1.40 \%\right)$.

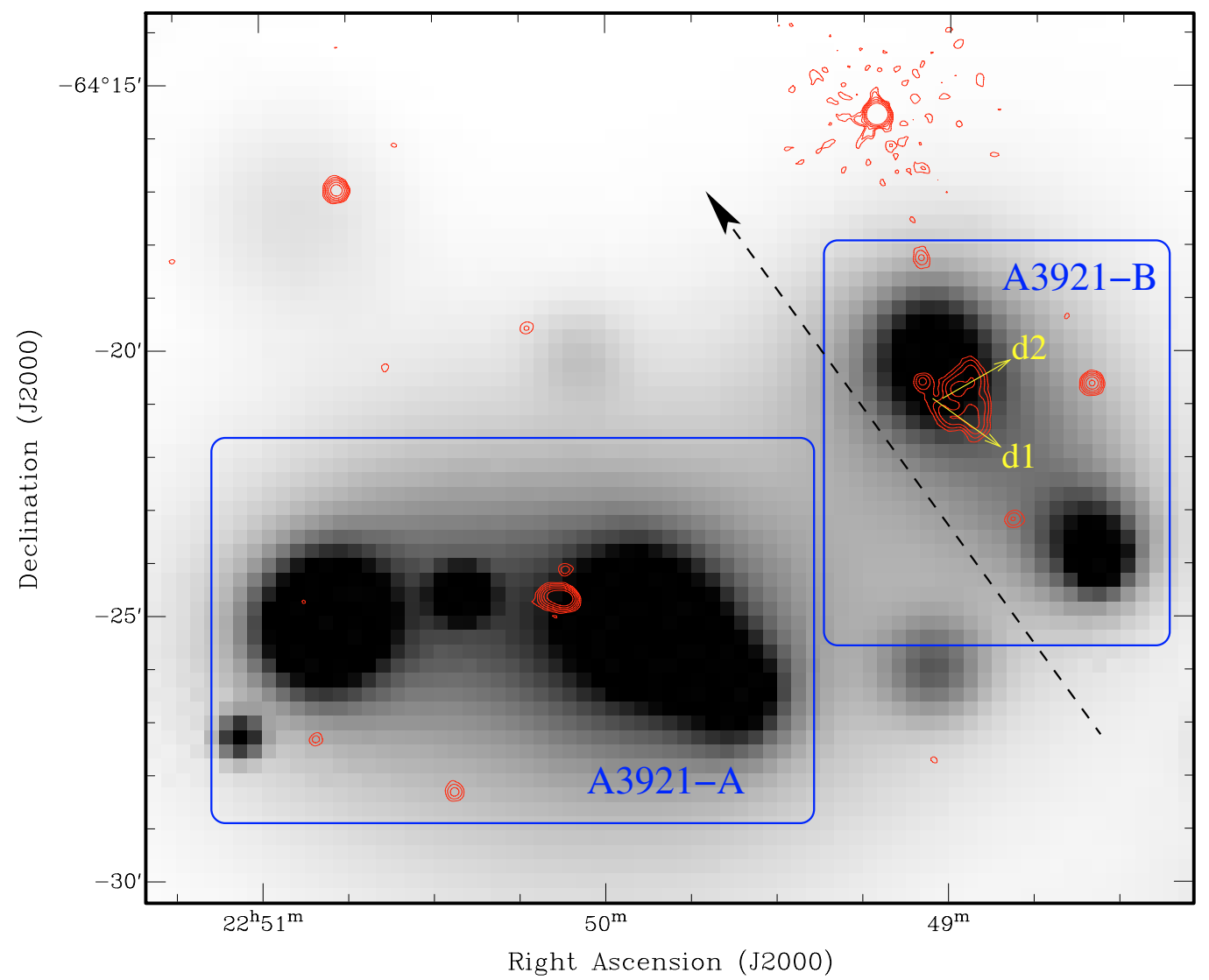

Fig. 7. $1.344 \mathrm{GHz}$ radio contours (levels from 0.294 to $15.680 \mathrm{mJy} /$ beam, with an increment factor of 2) overlaid on the projected galaxy density map of the galaxies lying in A3921 red sequence (F05). The two main sub-clusters of A3921 (A3921-A and A3921-B) and the main elongation axis of the radio tail of SUMSS J224857-642059 (R5) (d1 and d2) are indicated. The off-axis merging direction of A3921-B reconstructed from the combined optical and X-ray analyses is also shown with a dashed arrow (F05 and B05).

Kapferer et al. 2006). Our main aim was to closely investigate the possible connection between the on-going merging event and the SF properties of cluster members suggested by F05. We detected 17 radio sources, among which seven are cluster galaxies (confirmed or candidate). Based also on their optical and X-ray properties, the latter were classified as: a) two AGNs (R5 and R9), b) four late-type, gas rich galaxies (R2, R10, R11 and R14), and c) one (R3) strong star-burst. 


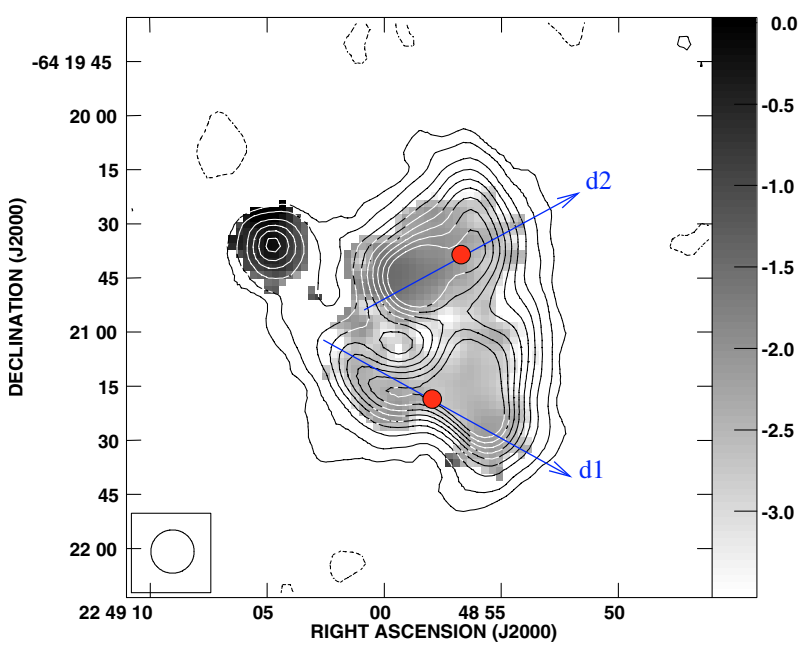

Fig. 8. Spectral index map of the radio source SUMSS J224857-642059 (R5) made by combining the images at $1.344 \mathrm{GHz}$ and $2.368 \mathrm{GHz}$. Contours are the same as in Fig. 6. The directions d1 and $\mathrm{d} 2$ along which the spectral index trend has been plotted in Fig. 9 are also shown. Circles give the position of the centre of the two slides of Fig. 9.

Seven radio emitting galaxies were therefore detected in the central field of A3921 at our $5 \sigma$ sensitivity level, of which four are late-type objects. In an equivalent cluster region and at the same flux limit (scaled to the proper $z$ ), 12 galaxies were detected at $1.4 \mathrm{GHz}$ both in A1367 and in Coma, of which nine and seven, respectively, have late-type morphologies (from Table 1 by Gavazzi \& Boselli 1999). Assuming Poissonian noise, we thus found a slight deficiency $(\sim 1 \sigma$ level) of radio galaxies in A3921 with respect to the lower- $z$ merging clusters Coma and A1367.

Among the 11 emission-line galaxies identified by F05, only two (the previously mentioned R2 and R3) were detected at our $3 \sigma$ sensitivity level $\left(\log \left(L_{22} \mathrm{~cm}(\mathrm{~W} / \mathrm{Hz})\right) \sim 21.4\right.$. The remaining nine objects are located in the collision region of the merging cluster. The lack of radio emission gave us an upper limit for their SFR unbiased by dust extinction $(S F R \lesssim$ $3 M_{\odot} \mathrm{yr}^{-1}$ ). Based on their observational properties (optical and radio luminosity, colour, presence and strength of emission lines in their spectra, SFR) and on their position in the cluster, we thus suggested that most of the emission-line galaxies detected in the collision region of A3921 are not infalling, gas-rich objects. Their SF episode would instead be related to the on-going sub-cluster collision. The merging event could have favoured both ICM-galaxy and tidal (galaxy-galaxy and cluster-galaxy) interactions, which have been shown to be able to trigger SF (e.g. Evrard 1991; Moss \& Whittle 2000; Poggianti et al. 2004; Bekki 1999; Kapferer et al. 2005; Boselli \& Gavazzi 2006). The cluster merger is probably also responsible for the strong starburst in R3 (BG3 in F05 and B05): the compression of the ICM on the ISM of this object is probably very strong, due both to the high ICM density (it is located at the X-ray centre of A3921-B, $\mathrm{B} 05$ ) and to the high proper velocity of this object (F05).

Note that, excluding R3, we did not detect any gas-rich, starforming galaxy in the collision region (Fig. 1 and Table 5). As summarised at the beginning of this Sect. (point b)), four gasrich, star-forming galaxies have been detected through our radio observations. All of them are located in the external parts of the merging sub-clusters, and they probably are infalling, field
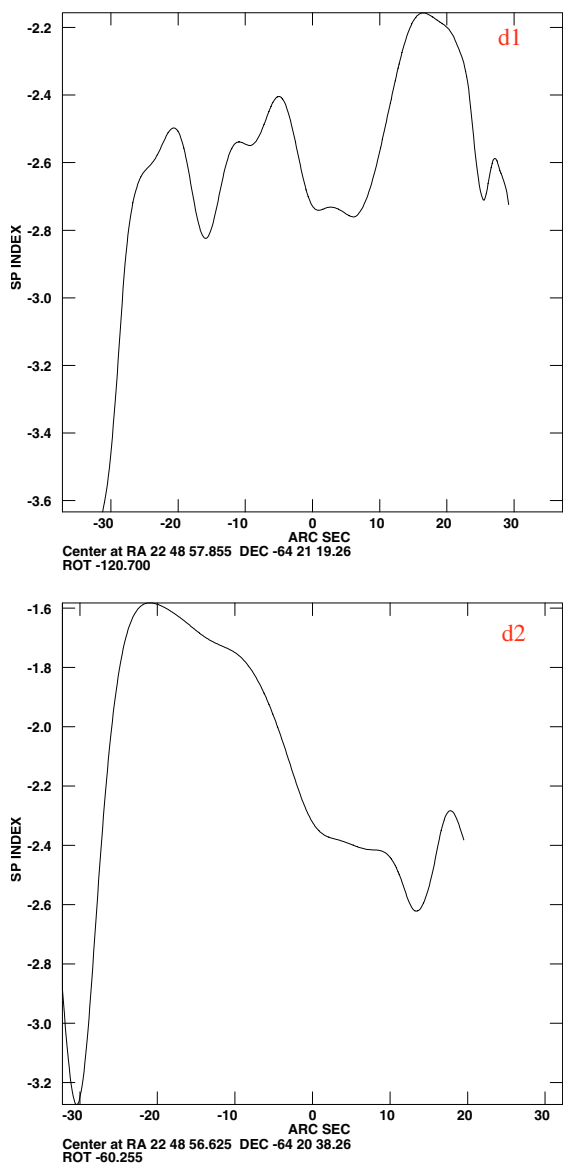

Fig. 9. Point-to-point spectral index between 1.344 and $2.368 \mathrm{GHz}$ along the main axis of SUMSS J224857-642059 (R5) tails (d1 and d2 in Fig. 8).

Table 6. Properties of the radio galaxy SUMSS J224857-642059 (R5).

\begin{tabular}{ll}
\hline \hline Core RA $\mathrm{R}_{\mathrm{J} 2000}$ & $22^{\mathrm{h}} 49^{\mathrm{m}} 04^{\mathrm{s}} .7$ \\
Core Dec $\mathrm{J}_{\mathrm{J} 000}$ & $-64^{\circ} 20^{\prime} 36^{\prime \prime} 6$ \\
Optical id. RA $\mathrm{J}_{\mathrm{J} 2000}$ & $22^{\mathrm{h}} 49^{\mathrm{m}} 04^{5} .8$ \\
Optical id. Dec $\mathrm{J}_{\mathrm{J} 000}$ & $-64^{\circ} 20^{\prime} 35^{\prime \prime} .6$ \\
Angular size $(1.344 \mathrm{GHz})$ & $\sim 105^{\prime \prime} \times 100^{\prime \prime}$ \\
Physical size $(1.344 \mathrm{GHz})$ & $\sim 170 \times 160 h_{75}{ }^{-1} \mathrm{kpc}$ \\
Flux density $(1.344 \mathrm{GHz})$ & $43.7 \mathrm{mJy}$ \\
Power $(1.344 \mathrm{GHz})$ & $8.5 \times 10^{23} h_{75}{ }^{-2} \mathrm{~W} / \mathrm{Hz}$ \\
$\langle R M\rangle$ & $22.5 \mathrm{rad} / \mathrm{m}^{2}$ \\
$\sigma_{R M}$ & $4.8 \mathrm{rad} / \mathrm{m}^{2}$ \\
$\left|R M_{\max }\right|$ & $33.5 \mathrm{rad} / \mathrm{m}^{2}$ \\
$\mathrm{H}_{\mathrm{eq}}$ & $3.6 \mu \mathrm{G}$ \\
Age & $8.7 \times 10^{7} \mathrm{yr}$ \\
\hline
\end{tabular}

spirals, whose gas reservoir has not yet been emptied by cluster environmental effects (Dressler \& Gunn 1983).

The absence of radio emission in all the $\mathrm{k}+\mathrm{a}$ galaxies detected in A3921 confirms that they are not dusty starbursting objects. This result agree with the conclusion of F05 and of previous analyses (e.g. Duc et al. 2002) that $\mathrm{k}+\mathrm{a}$ objects are observed during a post-star-forming phase. It also confirms that, in the A3921 central field, star-forming objects are concentrated in the collision region.

Our counts of radio emitting elliptical galaxies are not significantly different from the general cluster RLF derived by Ledlow \& Owen (1996). Similar numbers of early-type objects with radio luminosity typical of AGNs were detected in two other 
low- $z$ clusters, i.e. Coma and A1367 (Gavazzi \& Boselli 1999). Analogous results were also found by Venturi et al. (2001) in the pre-merging cluster A3528, suggesting that, in their early stages, cluster mergers do not influence the emission from radio-loud AGNs.

\subsection{The peculiar radio galaxy SUMSS J224857-642059 (R5)}

In order to understand the nature of the radio galaxy SUMSS J224857-642059 (R5) and the origin of its peculiar morphology, we have to take into account the following observational evidence:

- the position of its possible core component, coincident at $\sim 1$ arcsec uncertainty with the position of the optical galaxy BG2 (see Tables 4 and 6);

- its location at the centre of the less massive of two merging sub-clusters (A3921-B);

- its distorted tailed radio emission, without collimated jets, and roughly aligned with the merging axis (SW/NE). A NW elongation is, however, also present, and this shows a high polarized fraction and a nearly transverse intrinsic magnetic field;

- its steep total spectral index $\left(\left\langle\alpha_{1.344}^{2.368}\right\rangle=-2.4\right)$, typical of tailed radio sources in clusters of galaxies;

- the significantly flatter spectrum of the component associated with BG2 with respect to the extended emission $(\langle\alpha\rangle=$ -0.4 and $\langle\alpha\rangle=-2.6)$.

We therefore consider that R5 is a NAT radio source associated with BG2, with a core component in its Northernmost side, and an extended $\left(\sim 170 \times 160 h_{75}^{-1} \mathrm{kpc}\right)$ tail. This latter is distorted towards NW, possibly due to a lower local density of the intracluster gas revealed by the XMM observations of B05. The tail morphology is very likely explained by ram-pressure effects due to the relative motion between the ICM and $\mathrm{BG} 2$, in turn related to the merging event of the host sub-cluster (A3921-B). The total radio power of $\mathrm{R} 5$ at $1.344 \mathrm{GHz}$ is $\log (P(\mathrm{~W} / \mathrm{Hz}))=24.0$. Its total flux density is dominated by the extended emission located outside the optical galaxy, with a flux density ratio of $\sim 18$ between the extended and the core component ( $\sim 41.4$ and $\sim 2.3$ mJy respectively).

$\mathrm{R} 5$ does not show visible jets and the emission in the tail is very steep ( $\alpha \simeq-2 \div-3.5$ ), suggesting that electrons are suffering strong radiation losses. The diffuse component of R5 would therefore be a "dying" tail, which has been partly detached from an earlier period of activity of the BG2 radio galaxy and whose final evolution is dominated by synchrotron losses. The extended emission is now presumably supported and distorted by the ICM pressure. The strong polarization at the extreme of the NW component of the tail suggests that the radio jet has encountered a denser (or higher pressure) region leading to magnetic field compression.

Acknowledgements. C.F. is very grateful to Lister Staveley-Smith for his valuable help in ATCA data reduction. We warmly thank Vincent McIntyre for having provided the new version of the MIRIAD script "quickfit". We thank Christophe Benoist, Wolfgang Kapferer, Rocco Piffaretti, Alberto Cappi, PierreAlain Duc, Elaine Sadler and Jean-Luc Sauvageot for very fruitful discussions. We are indebted to the referee, Giuseppe Gavazzi, for useful suggestions which helped to improve the paper. C.F. acknowledges financial support of Sydney University, Australia Telescope National Facility, Marie Curie individual fellowship MEIF-CT-2003-900773, Austrian Science Foundation (FWF) through grant number P15868, and Tiroler Wissenschaftsfonds (TWF). The Australia Telescope Compact Array is part of the Australia Telescope which is funded by the Commonwealth of Australia for operation as a National Facility managed by CSIRO.

\section{References}

Arnaud, M., Rothenflug, R., Böhringer, H., Neumann, D., \& Yamashita, K. 1996, uxsa. coll., 163

Baldi, A., Bardelli, S., \& Zucca, E. 2001, MNRAS, 324, 509

Bekki, K. 1999, ApJ., 510, L15

Belsole, E., Sauvageot, J.-L., Pratt, G. W., \& Bourdin, H. 2005, A\&A, 430, 385 (B05)

Blandford, R. D., \& Rees, M. J. 1974, MNRAS, 169, 395

Boselli, A., \& Gavazzi, G. 2006, PASP, 118, 517

Butcher, H., \& Oemler, A. 1978, ApJ, 219, 18

Caldwell, N., Rose, J. A., Sharples, R. M., Ellis, R. S., \& Bower, R. G. 1993, AJ, 106,473

Coia, D., McBreen, B., Metcalfe, L., et al. 2005, A\&A, 431, 433

Condon, J. J. 1992, ARA\&A, 30, 575

Condon, J. J. 1997, PASP, 109, 166

Dallacasa, D., Feretti, L., Giovannini, G., \& Venturi, T. 1989, A\&AS, 79, 391

Dressler, A. 1987, in Nearly Normal Galaxies: From the Planck Time to the Present, ed. S. Faber (New York: Springer), 276

Dressler, A., \& Gunn, J. E. 1983, ApJ, 270, 7

Dressler, A., Smail, I., Poggianti, B. M., et al. 1999, ApJ, 122, 51

Duc, P. A., Poggianti, B. M., Fadda, D., et al. 2002, A\&A, 382, 60

Dwarakanath, K. S., \& Owen, F. N. 1999, AJ, 118, 625

Evrard, A. E. 1991, MNRAS, 248, 8

Feretti, L., Giovannini, G., Klein, U., et al. 1998, A\&A, 331, 475

Ferrari, C., Benoist, C., Maurogordato, S., Cappi, A., \& Slezak, E. 2005, A\&A, 430, 19 (F05)

Fujita, Y., Takizawa, M., Nagashima, M., \& Enoki, M. 1999, PASJ, 51, L1

Gavazzi, G., \& Boselli, A. 1999, A\&A, 343, 93

Gavazzi, G., Contursi, A., Carrasco, L., et al. 1995, A\&A, 304, 325

Gavazzi, G., Cortese, L., Boselli, A., et al. 2003, ApJ, 597, 210

Giacintucci, S., Venturi, T., Bardelli, S., Dallacasa, D., \& Zucca, E. 2004, A\&A, 419, 71

Goto, T. 2004, A\&A, 427, 125

Gregorini, L., \& Bondi, M. 1989, A\&A, 225, 333

Hopkins, A. M., Afonso, J., Chan, B., et al. 2003, AJ, 125, 465

Kapferer, W., Ferrari, C., Domainko, W., et al. 2006, A\&A, 447, 827

Kapferer, W., Knapp, A., Schindler, S., Kimeswenger, S., \& van Kampen, E. 2005, A\&A, 438, 87

Kennicutt, R. C. 1992, ApJ, 388, 310

Kennicutt, R. C. 1998, ARA\&A, 36, 189

Katgert, P., Mazure, A., den Hartog, R., et al. 1998, A\&AS, 129, 399

Katgert, P., Mazure, A., Perea, J., et al. 1996, A\&A, 310, 8

Ledlow, M. J., \& Owen, F. N. 1996, AJ, 112, 9

Mazure, A., Katgert, P., den Hartog, R., et al. 1996, A\&A, 310, 31

Miller, N. A., Owen, F. N., \& Hill, J. M. 2003, AJ, 125, 2393

Miller, N. A., \& Owen, F. N. 2002, AJ, 124, 2453

Morrison, G. E., \& Owen, F. N. 2003, AJ, 125, 506

Moss, C., \& Whittle, M. 2000, MNRAS, 317, 667

Murgia, M., Parma, P., de Ruiter, H. R., Mack, K.-H., \& Fanti, R. 2004, in X-Ray and Radio Connections, ed. L. O. Sjouwerman, \& K. K. Dyer, http://www.aoc.nrao.edu/events/xraydio/ [meetingcont/8.15_murgia.pdf]

Owen, F. N., Keel, W. C., Ledlow, M. J., Morrison, G. E., \& Windhorst, R. A. 2005a, AJ, 129, 26

Owen, F. N., Ledlow, M. J., Keel, W. C., Wang, Q. D., \& Morrison, G. E. 2005b, AJ, 129, 31

Owen, F. N., Ledlow, M. J., Keel, W. C., \& Morrison, G. E. 1999, AJ, 118, 633

Petrosian, V., \& Dickey, J. 1973, ApJ, 186, 403

Poggianti, B. M., \& Wu, H. 2000, ApJ, 529, 157

Poggianti, B. M., Smail, I., Dressler, A., et al. 1999, ApJ, 518, 576

Poggianti, B. M., Bridges, T. J., Komiyama, Y., et al. 2004, ApJ, 601, 197

Rizza, E., Morrison, G. E., Owen, F. N., et al. 2003, AJ, 126, 119

Sault, R. J., Staveley-Smith, L., \& Brouw, W. N. 1996, A\&AS, 120, 375

Serjeant, S., Gruppioni, C., \& Oliver, S. 2002, MNRAS, 330, 621

Simard-Normandin, M., Kronberg, P. P., \& Button, S. 1981, ApJS, 45, 97

Smail, I., Morrison, G., Gray, M. E., et al. 1999, ApJ, 525, 609

Venturi, T., Bardelli, S., Morganti, R., \& Hunstead, R. W. 2000, MNRAS, 314, 594

Venturi, T., Bardelli, S., Zambelli, G., Morganti, R., \& Hunstead, R. W. 2001, MNRAS, 324, 1131

Venturi, T., Bardelli, S., Zagaria, M., Prandoni, I., \& Morganti, R. 2002, A\&A, 385, 39

Wolf, C., Gray, M. E., \& Meisenheimer, K. 2005, A\&A, 443, 435 